\title{
Consistency of Open-Loop Experimental Frequency- Response Data with Coprime Factor Plant Models
}

\author{
Benoit Boulet, Member, IEEE, and Bruce A. Francis, Fellow, IEEE
}

\begin{abstract}
The model/data consistency problem for coprime factorizations considered here is this: Given some possibly noisy frequency-response data obtained by running open-loop experiments on a system, show that these data are consistent with a given family of perturbed coprime factor models and a timedomain $\mathcal{L}_{\infty}$ noise model. In the noise-free open-loop case, the model/data consistency problem boils down to the existence of an interpolating function in $\mathcal{R} \mathcal{H}_{\infty}$ that evaluates to a finite number of complex matrices at a finite number of points on the imaginary axis. A theorem on boundary interpolation in $\mathcal{R} \mathcal{H}_{\infty}$ is a building block that allows us to devise computationally simple necessary and sufficient tests to check if the perturbed coprime factorization is consistent with the data. For standard coprime factorizations, the test involves the computation of minimum-norm solutions to underdetermined complex matrix equations. The Schmidt-Mirsky theorem is used in the case of special factorizations of flexible systems. For $\mathcal{L}_{\infty}$ noise corrupting the frequency-response measurements, a complete solution to the open-loop noisy single-input/single-output problem using the structured singular value $\mu$ is given.
\end{abstract}

Index Terms-Coprime factorization, model validation, Nevanlinna-Pick interpolation, robustness, uncertain systems.

\section{INTRODUCTION}

$\mathbf{T}$ HIS PAPER addresses the problem of checking consistency of open-loop experimental frequency-response data with perturbed coprime factor plant descriptions. This issue has been largely overlooked in the development of robust control, although some recent work has dealt with this problem for time-response data [15], [16], [20], [22]. This is somewhat surprising because one might argue that the problem of uncertainty modeling should not be determined only by how tractable the robustness problem gets with a given model, but also from the possibility of performing experimental consistency checks and by the feasibility of constructing bounds for the uncertainty sets from experimental data. Related works on identification in $\mathcal{H}_{\infty}$ use frequencyresponse data [3], [7], [12], and in particular, [7] and [12] use the Nevanlinna-Pick interpolation theory as we do.

Manuscript received January 24, 1997; revised December 26, 1997. Recommended by Associate Editor, H. Ozbay. This work was supported by scholarships from the Natural Sciences and Engineering Research Council (NSERC) of Canada, the Fonds pour la formation des chercheurs et l'aide à la recherche, Québec, and a Walter C. Sumner Memorial Fellowship.

B. Boulet is with the Systems and Control Group and Centre for Intelligent Machines, Department of Electrical and Computer Engineering, McGill University, Montréal, Québec H3A 2A7 Canada (e-mail: boulet@cim.mcgill.ca).

B. A. Francis is with Systems Control Group, Department of Electrical and Computer Engineering, University of Toronto, Toronto, Ontario, M5S 1A4 Canada.

Publisher Item Identifier S 0018-9286(98)09438-0.
The general model/data consistency problem for coprime factorizations $(\mathrm{CF})$ is this: Given some experimental data obtained by running open-loop or closed-loop experiments on the system, show that the (possibly noisy) data are consistent with a family of perturbed coprime factor models. In other words, do there exist a perturbation and a noise belonging to their respective sets such that the input-output data can be reproduced by the model? A mathematical formulation of this problem is given in the next section.

For frequency-domain noisy input-output data, Smith and Doyle [22] developed a method based on optimization to answer this question. Their method applies to a general uncertainty description, where the uncertainty block is connected as a feedback around a generalized plant. Since coprime factor uncertainty fits this description, the method can be applied to the special case of $\mathrm{CF}$ which we are interested in. The noise is treated as an exogenous input of the generalized plant and is assumed to be bounded in $\mathcal{L}_{2}$. More recently, Chen [6] provided solutions to the general model/data consistency problem in the frequency domain for uncertain discrete-time plants represented by linear fractional transformations (LFT's), with structured and unstructured uncertainty. Two frequencydomain problems were considered in [6], namely checking consistency using multivariable input-output data and using frequency samples of the plant. The work of [22] provided solutions for the former problem, whereas in this paper, we are concerned with the latter problem for continuous-time systems with coprime factor models. In [6], Chen showed that these general consistency problems can be transformed into a set of convex optimization problems, which can then be solved using convex optimization techniques. The theoretical results of [6] are quite general, albeit not straightforward in their application, a comment that also applies to [22]. Our results for the special case of coprime factorizations are arguably easier to apply, since one only needs to compute standard or structured singular values of constant matrices for consistency checks.

We consider the case where we have $N$ frequency-response data points to test the model. The open-loop noise-free multiinput/multi-output (MIMO) case and noisy single-input/singleoutput (SISO) case are studied, and complete solutions to these consistency problems are given. One motivation for using a coprime factorization approach is that it is well suited for modeling low-damped dynamics of large flexible space structures (LFSS) [5] or flexible mechanical systems with clustered modes. If frequency-response experiments are performed on an experimental setup with flexibilities or on an actual LFSS, then the results presented in this paper are of interest to obtain better uncertainty models. 
The open-loop noise-free model/data consistency problem boils down to the existence of an interpolating function in $\mathcal{R} \mathcal{H}_{\infty}$ that evaluates to a finite number $N$ of complex matrices of compatible dimensions at $N$ distinct points on the imaginary axis. Our central result is a theorem on boundary interpolation in $\mathcal{R} \mathcal{H}_{\infty}$. This necessary and sufficient condition allows us to devise a simple test consisting of computing minimumnorm solutions to $N$ underdetermined linear complex matrix equations to check if the perturbed coprime factorization is consistent with the data. Left-coprime factorizations (LCF) are studied, but the results also apply to right-coprime factorizations (RCF). We also treat the case of a special factorization for LFSS's introduced in [5]. This case turns out to be quite a bit more difficult than the standard coprime factorization case, but a complete solution is nonetheless provided. This necessary and sufficient condition uses the Schmidt-Mirsky theorem [23, Th. 4.18] and involves the computation of the $p$ th singular value of a complex matrix for each measurement frequency.

Then the open-loop noisy SISO model/data consistency problem is considered for time-domain bounded $\mathcal{L}_{\infty}$ noise corrupting the measured output sinusoids in frequency-response measurements. An approximation of the effect of such noises in the frequency-domain is disk-like uncertainty in the complex plane around each datum of the complex frequency response for each of $N$ distinct frequencies. So the problem is again decomposed into $N$ consistency problems for constant complex matrices, and then the boundary interpolation theorem is invoked to show that there exists (or not) a norm-bounded $\mathcal{R} \mathcal{H}_{\infty}$ perturbation of the coprime factors that could have produced the noisy frequency-response measurements. For the SISO case considered, $N \mu$ tests on linear fractional transformations provide a necessary and sufficient condition for consistency.

We show that if some of the singular value tests are negative, then the norm bound on the coprime factor uncertainty model can be increased at the corresponding frequencies until all $N$ tests lead to a positive conclusion of consistency of the model with the data. On the other hand, it is also possible to reduce the norm bound at those frequencies where the tests show that there is too much uncertainty in the model. This provides a way to refine the model by reducing conservativeness at some frequencies while ensuring consistency for all measurements.

\section{A. Notation}

Let $H$ be an $n \times m$ complex matrix with singular values $\sigma_{1} \geq \cdots \geq \sigma_{q}, q:=\min \{m, n\}$. The maximum and minimum singular values of $H$ are written as $\bar{\sigma}(H)=\sigma_{1}$ and $\underline{\sigma}(H)=\sigma_{q}$, respectively. The norm of $H$ is taken to be its maximum singular value $\|H\|=\bar{\sigma}(H)$, and $H^{*}$ is its conjugate transpose. We denote the open and closed right-half complex planes by $C_{+}$and $\bar{C}_{+}$, respectively. The extension of $\bar{C}_{+}$to infinity is written as $\bar{C}_{+} \cup\{\infty\}$. The open unit disk in $C$ is denoted as $D$. The space $\mathcal{H}_{\infty}$ is the class of functions analytic in $C_{+}$and bounded on the imaginary axis with norm defined as $\|Q\|_{\infty}=\sup _{\omega \in R}\|Q(j \omega)\|$. The space $\mathcal{H}_{\infty}(D)$ is the class of functions analytic and bounded in $D$ with norm defined as $\|\mathbf{G}\|_{\infty}:=\sup _{\theta \in[0,2 \pi)}\left\|\mathbf{G}\left(e^{j \theta}\right)\right\|$. The prefix $\mathcal{R}$ on $\mathcal{R} \mathcal{H}_{\infty}$ denotes real-rational; thus $\mathcal{R} \mathcal{H}_{\infty}$ is the class of scalar or matrix-valued proper stable real-rational transfer functions. According to the context, it should be clear whether we are considering scalar or matrix-valued functions, but sometimes we will write, say, $\mathcal{H}_{\infty}^{m \times n}$. For a normed space $\mathcal{X}, \mathcal{B X}$ denotes its open unit ball. A function in $\mathcal{H}_{\infty}\left(\mathcal{R} \mathcal{H}_{\infty}\right)$ is a unit if its inverse also belongs to $\mathcal{H}_{\infty}\left(\mathcal{R} \mathcal{H}_{\infty}\right)$. Signals are represented with lowercase letters and their Laplace transforms are just the same letters with hats. For finite-dimensional linear time-invariant operators, scalar constants and functions are represented respectively by regular and boldface lowercase letters, while matrix constants and matrix-valued functions are assigned respectively regular and boldface uppercase letters. Upper and lower LFT's are now defined. Suppose the matrix $P$ is partitioned as

$$
P:=\left[\begin{array}{ll}
P_{11} & P_{12} \\
P_{21} & P_{22}
\end{array}\right]
$$

$K$ is such that its transpose $K^{T}$ has the same dimensions as $P_{22}$, and $\Delta$ is such that its transpose $\Delta^{T}$ has the same dimensions as $P_{11}$. Then the lower LFT of $P$ by $K$ is $\mathcal{F}_{L}(P, K):=P_{11}+P_{12} K\left(I-P_{22} K\right)^{-1} P_{21}$ whenever the inverse exists. The upper LFT of $P$ by $\Delta$ is $\mathcal{F}_{U}(P, \Delta):=$ $P_{22}+P_{21} \Delta\left(I-P_{11} \Delta\right)^{-1} P_{12}$ whenever the inverse exists.

\section{Problem Formulation}

This section introduces a general model/data consistency problem and then specializes it to coprime factorizations. The formulation includes as special cases all the problems considered in this paper. Define an experiment as follows: Apply an input to the system, and record the input and the resulting output. The general model/data consistency problem is comprised of: 1) a set $\mathcal{M}$ of $N$ experiments; 2) a set $\mathcal{W}$ of noise signals; 3 ) a nominal model $\mathbf{T}$; and 4) an uncertainty set $\mathcal{D}$. The noise affects the measurements in a specified way, and uncertainty in the plant model has a specified structure (e.g., additive, multiplicative, etc.) Note that the experiments can be open-loop or closed-loop, and hence $\mathbf{T}$ may be an open-loop or a closed-loop model. The general problem is formulated as follows.

Problem 1: Given the sets $\mathcal{M}, \mathcal{W}, \mathcal{D}$ and a nominal model $\mathbf{T}$, do there exist a perturbation $\boldsymbol{\Delta} \in \mathcal{D}$ of $\mathbf{T}$ and a subset $\mathcal{V} \subset \mathcal{W}$ of $N$ noise signals, one for each experiment, such that the experiments $\mathcal{M}$ can be reproduced with $\mathbf{T}$ perturbed by $\Delta$ together with the noises $\mathcal{V}$ ?

Note that this problem is weaker than the model validation problem where one seeks to validate the model triple $(\mathbf{T}, \mathcal{D}, \mathcal{W})$ using the measurements $\mathcal{M}$. As a matter of fact, this latter problem was abandoned when Popper [21] showed that a model can never be validated using a finite set of data. The best one can do is to show that the model triple $(\mathbf{T}, \mathcal{D}, \mathcal{W})$ is not invalidated by the experimental data $\mathcal{M}$, and this is what the model/data consistency problem is all about [20], [22]. A negative answer to Problem 1 forces us to modify $\mathbf{T}$ and/or $\mathcal{D}$ until we get consistency. In that sense, our knowledge of the system increases. 
Let us now specialize Problem 1 to finite-dimensional linear time-invariant $\mathbf{T}$, which would be the plant model $\mathbf{G}$ of which we have a coprime factorization in $\mathcal{R} \mathcal{H}_{\infty}$ in the open-loop case. The data set $\mathcal{M}$ is a set of $N$ complex matrices (or complex scalars for SISO systems) obtained by running open-loop frequency-response experiments at $N$ distinct frequencies. Each entry $(i, j)$ of one of those matrices is a complex number whose magnitude and phase represent, respectively, the gain and phase of the plant from the $j$ th input to the $i$ th output at the corresponding frequency. The noise set $\mathcal{W}$ is composed of complex matrices with magnitudebounded entries, of the same dimensions as the plant model. Each entry $(i, j)$ of a matrix in $\mathcal{W}$ represents a complex additive perturbation of the corresponding entry of the nominal frequency response matrix $\mathbf{T}(j \omega)$ at frequency $\omega$. Finally, the uncertainty set $\mathcal{D}$ is composed of norm-bounded factor perturbations in $\mathcal{R} \mathcal{H}_{\infty}$.

Let the nominal plant model $\mathbf{G}$ be a stable proper realrational transfer matrix. Let the square, invertible $\tilde{\mathbf{M}}$ and $\tilde{\mathbf{N}}$ in $\mathcal{R H}_{\infty}$ be left-coprime, $\mathbf{C}$ be an output transfer matrix, and $\mathbf{J}$ be a diagonal input transfer matrix, both in $\mathcal{R} \mathcal{H}_{\infty}$, such that the nominal plant model can be factorized as $\mathbf{G}=\mathbf{C M}^{-1} \tilde{\mathbf{N}} \mathbf{J}$. The matrices $\mathbf{C}$ and $\mathbf{J}$ are included for compatibility with the special factorization for LFSS's introduced in [5]. When set to identity matrices, a standard LCF of $\mathbf{G}$ is obtained. Let the perturbed plant model $\mathbf{G}_{p}$ be expressed as a perturbed factorization with $\tilde{\mathbf{M}}_{p}, \tilde{\mathbf{N}}_{p} \in \mathcal{R} \mathcal{H}_{\infty}$

$$
\mathbf{G}_{p}=\mathbf{C M}_{p}^{-1} \tilde{\mathbf{N}}_{p} \mathbf{J}
$$

where $\tilde{\mathbf{M}}_{p}=\tilde{\mathbf{M}}+\boldsymbol{\Delta}_{M}, \tilde{\mathbf{N}}_{p}=\tilde{\mathbf{N}}+\boldsymbol{\Delta}_{N}, \boldsymbol{\Delta}_{M}, \boldsymbol{\Delta}_{N} \in \mathcal{R} \mathcal{H}_{\infty}$. Define the uncertainty matrix $\boldsymbol{\Delta}:=\left[\boldsymbol{\Delta}_{N}-\boldsymbol{\Delta}_{M}\right]$. Clearly, $\boldsymbol{\Delta} \in \mathcal{R} \mathcal{H}_{\infty}$. This matrix is introduced because the result on robust closed-loop internal stability of the system (see, e.g., [11] for a definition and treatment of internal stability) in Fig. 1 for a perturbed coprime factor plant description is expressed in terms of a norm bound on $\boldsymbol{\Delta}(j \omega)$ [17], [24]. Define the uncertainty set

$$
\mathcal{D}_{r}:=\left\{\boldsymbol{\Delta} \in \mathcal{R} \mathcal{H}_{\infty}:\left\|\mathbf{r}^{-1} \boldsymbol{\Delta}\right\|_{\infty}<1\right\}
$$

and the family of plants

$$
\mathcal{P}:=\left\{\mathbf{G}_{p}: \Delta \in \mathcal{D}_{r}\right\}
$$

where $\mathrm{r}$ is a unit in $\mathcal{R \mathcal { H }}_{\infty}$. For the open-loop case treated in this paper, $\mathbf{r}$ is assumed small enough so that $\tilde{\mathbf{M}}_{p}$ is also a unit in $\mathcal{R H}_{\infty}$, that is, every $\mathbf{G}_{p} \in \mathcal{P}$ is stable. For this to hold, we shall make the following assumption.

$$
\text { A1) } \underline{\sigma}[\tilde{\mathbf{M}}(j \omega)]>|\mathbf{r}(j \omega)|, \forall \omega \in R \cup\{\infty\} \text {. }
$$

The unit $\mathbf{r}$ characterizes the size of the uncertainty in the coprime factors at each frequency $\omega$ because $\left\|\mathbf{r}^{-1} \boldsymbol{\Delta}\right\|_{\infty}<1$ implies $\|\boldsymbol{\Delta}(j \omega)\|<|\mathbf{r}(j \omega)|$. The small-gain theorem yields the following slightly modified result of [24] (see also [17]).

Theorem 1: The closed-loop system of Fig. 1 with controller $\mathbf{K}$ is internally stable for every $\mathbf{G}_{p} \in \mathcal{P}$ iff:

1) $\mathbf{K}$ internally stabilizes $\mathbf{G}$;

2) $\left\|\mathrm{r}\left[\begin{array}{c}\mathbf{J K C}(I-\mathbf{G J K C})^{-1} \tilde{\mathbf{M}}^{-1} \\ (I-\mathbf{G J K C})^{-1} \tilde{\mathbf{M}}^{-1}\end{array}\right]\right\|_{\infty} \leq 1$.

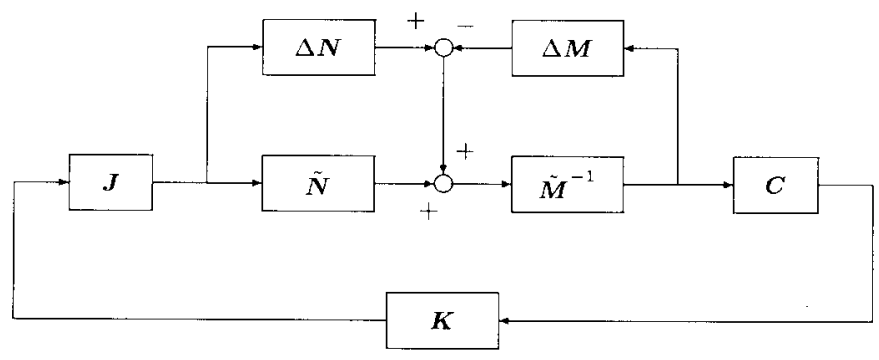

Fig. 1. Feedback control of a perturbed LCF model.

Theorem 1 on robust stability of a closed-loop system with a perturbed coprime factorization as in Fig. 1 gives the main motivation to make $\mathbf{r}$ as small as possible.

In order to be able to use the result of Theorem 1 in the design of a robust controller for a real plant, one has to construct and modify the bound $|\mathrm{r}(j \omega)|$ until it properly captures the uncertainty in the physical system. One way to do this is to start with a nominal model and a first approximation for $\mathbf{r}$, and then use experimental data to check if $|\mathbf{r}(j \omega)|$ is large enough to account for the full data set. It can be made larger if necessary, but not too large to avoid making the model too conservative. It can also be made smaller to reduce the uncertainty at some frequencies. The consistency tests proposed in this paper are suitable for that purpose when open-loop frequency-response data are available.

Note that it is assumed throughout that the plant is linear. We will consider the case of time-domain $\mathcal{L}_{\infty}$-bounded output noises corrupting the frequency-response measurements and show that their effects can be modeled as complex noises in the frequency domain, i.e., the noise set $\mathcal{W}$. Mild nonlinearities or time-variances which would have an effect comparable to $\mathcal{L}_{\infty}$ noise in the time domain (such as a bias) cannot be detected, so checking consistency of the data with a linear model would be feasible in this case. But the interpretation and implications of the results are not studied here. However, an exception to this is the case of a nonlinearity at the output of an openloop plant, which would have the same effect as additive $\mathcal{L}_{\infty}$-bounded output noise. For example, a quantizer at the output can be modeled as an additive output noise bounded in $\mathcal{L}_{\infty}$ and corrupting the output signal of a linear plant. In this case, the theory developed in this paper is applicable.

\section{THE NoISE MODEL}

The goal of this section is to show that time-domain $\mathcal{L}_{\infty^{-}}$ bounded additive output noise corrupting a frequency-response measurement at frequency $\omega$ can be modeled as a complex noise added to $\mathbf{T}(j \omega)$.

A frequency-response experiment consists of exciting the system with a sine wave of a given frequency $\omega$ and recording the time-response of the output after the transients have died down. The input sinusoid is assumed to have unit amplitude. The noise affects magnitude and zero-crossing readings on the recorded output signal as shown in Fig. 2. We assume that the noise is any signal in $\mathcal{L}_{\infty}$ bounded in magnitude; thus the noise set $\mathcal{N}$ considered here is all scalar time-domain noises 


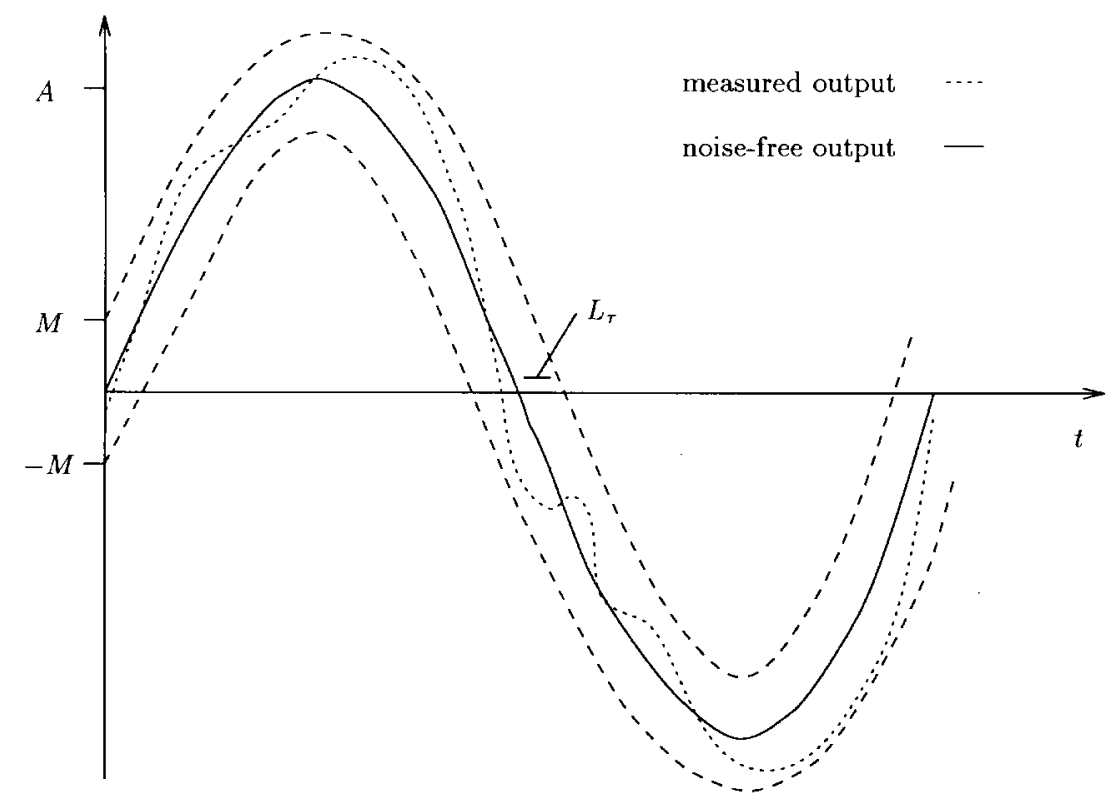

Fig. 2. Recorded noisy output signal.

in $\mathcal{L}_{\infty}$ with norm bounded by $M$, i.e.,

$$
\mathcal{N}:=\left\{n \in \mathcal{L}_{\infty}:\|n\|_{\infty}<M\right\}
$$

Let $A_{m}$ be the measured magnitude of the output signal and $\tau_{m}$ be any measured time-delay between the output signal and the input sinusoid at zero crossings. We have $A_{m}=A+w_{A}$ and $\tau_{m}=\tau+w_{\tau}$. The amplitude measurement noise is then bounded by $\left|w_{A}\right| \leq M$, and the phase measurement noise $w_{\theta}$ is derived by first characterizing the delay measurement noise $w_{\tau}$. A first-order approximation of the sine function around a zero crossing yields the bound $\left|w_{\tau}\right| \leq L_{\tau}$, where $L_{\tau}=\frac{M}{A \omega}$, which leads to the following bound on the phase measurement noise:

$$
\left|w_{\theta}\right| \leq \omega L_{\tau}=\frac{M}{A} \leq \frac{M}{A_{m}-M}=: L_{\theta} .
$$

The set $\mathcal{U}:=\left\{\left(A+w_{A}\right) e^{j\left(\theta+w_{\theta}\right)}:\left|w_{A}\right| \leq M,\left|w_{\theta}\right| \leq L_{\theta}\right\}$ in $C$ is approximated by the largest disk $\mathcal{W}_{0} \subset \mathcal{U}$. Thus, for SISO plants, the corresponding frequency-domain noise set $\mathcal{W}$ that appears in the specialized version of Problem 1 above is the set of additive norm-bounded complex noises

$$
\mathcal{W}:=\left\{w_{a} \in C:\left|w_{a}\right|<L_{a}\right\}
$$

where $L_{a}$ is the radius of $\mathcal{W}_{0}$ (which should be close to $M)$. Note that $\mathcal{W}$ is just the disk $\mathcal{W}_{0}$ translated to the origin. As examples, noises caused by external vibrations in a mechanical system, or electrical noises affecting the sensor may be bounded by a unique constant for all measurements. Quantization noise may also be bounded in this way. Note that the bound $L_{a}$ does not depend on the frequency $\omega$, nor on the amplitude of the output sinusoid, which is a nice property.

\section{CONSISTENCY WITH OPEN-LOOP FREQUENCY-RESPONSE MEASUREMENTS}

\section{A. Noise-Free Case}

Standard Factorization: Suppose we are given a family of $m$-input, $p$-output stable plants $\mathcal{P}$ as in (3) with $\mathbf{C}=I_{p}$ and $\mathbf{J}=I_{m}$, and a set of noise-free, open-loop frequencyresponse data. In this section, we derive a necessary and sufficient condition for consistency of these data with the family of plants $\mathcal{P}$. The exact statement of the open-loop noisefree model/data consistency problem considered here is the following.

Problem 2: Given noise-free, open-loop frequencyresponse data $\left\{\Phi_{i}\right\}_{i=1}^{N} \subset C^{p \times m}$ of an $m$-input, $p$-output plant obtained at the distinct frequencies $\omega_{1}, \cdots, \omega_{N}$, could the data have been produced by at least one model in $\mathcal{P}$ ? Or, in other words, does there exist a $\Delta \in \mathcal{D}_{r}$ such that the corresponding perturbed model $\mathbf{G}_{p}$ interpolates the complex matrices $\left\{\Phi_{i}\right\}_{i=1}^{N}$ at $j \omega_{1}, \cdots, j \omega_{N}$ ?

Premultiplying (1) by $\tilde{\mathbf{M}}+\boldsymbol{\Delta}_{M}$ and taking $\boldsymbol{\Delta}_{M}$ and $\boldsymbol{\Delta}_{N}$ onto the left-hand side, we get

$$
\boldsymbol{\Delta}_{M} \mathbf{G}_{p}-\boldsymbol{\Delta}_{N}=\tilde{\mathbf{N}}-\tilde{\mathbf{M}} \mathbf{G}_{p} \text {. }
$$

Let $U:=\left(\tilde{\mathbf{N}}-\tilde{\mathbf{M}} \mathbf{G}_{p}\right)(j \omega), W:=\left[\begin{array}{l}-I_{m} \\ -\mathbf{G}_{p}\end{array}\right](j \omega)$, and $\Delta:=$ $\Delta(j \omega)$. Then (6) at frequency $\omega$ can be written as

$$
\Delta W=U
$$

where $W \in C^{(m+p) \times m}, U \in C^{p \times m}$, and $\Delta \in C^{p \times(m+p)}$. Equation (7) is just an underdetermined system of linear equations over the field $C$.

Let $\Delta_{i}=\boldsymbol{\Delta}\left(j \omega_{i}\right)$ for $i=1, \cdots, N$, with similar definitions for $W_{i}$ and $U_{i}$. We seek a test that would show whether or not there exists a rational matrix $\boldsymbol{\Delta}$ that belongs to the uncertainty set $\mathcal{D}_{r}$ and satisfies the interpolation constraints given by (7) at the distinct frequencies $\left\{\omega_{1}, \cdots, \omega_{N}\right\}$. This is done in two 
steps: First, with $\mathbf{G}_{p}\left(j \omega_{i}\right)=\Phi_{i}$ solve the matrix equation (7) for $\Delta_{i}, i=1, \cdots, N$, such that $\Delta_{i}$ has minimum norm. Since $W_{i}$ has full column rank, the minimum-norm solution $\Delta_{i}$ to (7) is

$$
\Delta_{i}=U_{i}\left(W_{i}^{*} W_{i}\right)^{-1} W_{i}^{*} .
$$

If the norm of $\Delta_{i}$ is greater than or equal to $\left|\mathbf{r}\left(j \omega_{i}\right)\right|$ for some $i \in\{1, \cdots, N\}$, then the test fails: the family of coprime factorizations cannot account for the frequency-response data. If $\left\|\Delta_{i}\right\|<\left|\mathbf{r}\left(j \omega_{i}\right)\right|, \forall i \in\{1, \cdots, N\}$, then we must show that there exists a matrix-valued function $\Delta \in \mathcal{R} \mathcal{H}_{\infty}$ taking on the complex matrix values $\left\{\Delta_{i}\right\}_{i=1}^{N}$ at the frequencies $\left\{\omega_{i}\right\}_{i=1}^{N}$ and such that $\|\boldsymbol{\Delta}(j \omega)\|<|\mathbf{r}(j \omega)|, \forall \omega \in R$. Those are the two steps in the proof of the main result of this section, which goes as follows.

Theorem 2: The noise-free open-loop MIMO CF model/ data consistency problem (Problem 2) has a positive answer iff $\| \Delta_{i}||<\left|\mathbf{r}\left(j \omega_{i}\right)\right|$ for all $i=1, \cdots, N$.

This result, proven in Appendix A, draws on the boundary interpolation theorem, whose detailed proof is also provided in the Appendix. Note that Chen [6] also uses the boundary interpolation theorem for his consistency results, but he does not give a proof for it. The bound $|\mathrm{r}(j \omega)|$ can be shaped such that the inequality in the theorem statement is satisfied for all $i$. One can see how $\mathrm{r}$ can be constructed and improved as new experimental data become available.

Numerical Example: Suppose we are given a nominal coprime factorization $\mathbf{G}=\tilde{\mathbf{M}}^{-1} \tilde{\mathbf{N}}$, with

$$
\tilde{\mathbf{M}}=\left[\begin{array}{cc}
\frac{s+2}{s+1} & \frac{s+3}{s+2} \\
0 & \frac{s+1}{s+2}
\end{array}\right], \quad \tilde{\mathbf{N}}=\left[\begin{array}{ccc}
\frac{1}{s+1} & \frac{2}{s+1} & 0 \\
0 & \frac{1}{s+3} & \frac{2}{s+2}
\end{array}\right]
$$

and a bound on the factor perturbations $\mathbf{r}(s)=\frac{10^{-8} s+0.3}{s+1}$. With these, Assumption A1) holds. Consider the following coprime factorization of the "true" plant (unknown to us) $\mathbf{G}_{p}=\tilde{\mathbf{M}}_{p}^{-1} \tilde{\mathbf{N}}_{p}$, where

$$
\tilde{\mathbf{M}}_{p}=\left[\begin{array}{cc}
\frac{s+1.9}{s+1} & \frac{s+2.9}{s+2} \\
0.1 & \frac{1.1 s+1}{s+2}
\end{array}\right], \quad \tilde{\mathbf{N}}_{p}=\left[\begin{array}{ccc}
\frac{1.1}{s+1} & \frac{2.2}{s+1} & 0 \\
0 & \frac{0.9}{s+3} & \frac{1.8}{s+2}
\end{array}\right] \text {. }
$$

The factor perturbations are

$$
\boldsymbol{\Delta}_{M}=\left[\begin{array}{cc}
\frac{-0.1}{s+1} & \frac{-0.1}{s+2} \\
0.1 & \frac{0.1 s}{s+2}
\end{array}\right], \quad \boldsymbol{\Delta}_{N}=\left[\begin{array}{ccc}
\frac{0.1}{s+1} & \frac{0.2}{s+1} & 0 \\
0 & \frac{-0.1}{s+3} & \frac{-0.2}{s+2}
\end{array}\right] .
$$

Now suppose we take five frequency-response measurements $\left\{\Phi_{i}:=\mathbf{G}_{p}\left(j \omega_{i}\right)\right\}_{i=1}^{5}$ on the plant at the frequencies $\omega_{1}=0.01, \omega_{2}=0.1, \omega_{3}=1, \omega_{4}=10, \omega_{5}=100$ radians/s. Equation (8) is used repeatedly to compute the five 2 $\times 5$ minimum-norm perturbations solving the consistency (6) at the five measurement frequencies. Fig. 3 shows a plot of the magnitude of $\mathbf{r}(j \omega)$, the norm of $\boldsymbol{\Delta}(j \omega)$, and the norms of the $\Delta_{i}$ 's. The model is inconsistent with the data at the two frequencies $\omega_{4}$ and $\omega_{5}$, but it can be made consistent by simply shifting $|\mathbf{r}(j \omega)|$ toward higher frequencies. For instance, $\mathbf{r}_{1}(s)=\frac{10^{-8} s+0.3}{0.5 s+1}$ makes the model consistent with all the data.

Special Factorization for Square LFSS Models: Let us now consider a nominal factorization of a square openloop plant model $\mathbf{G}$ of the form $\mathbf{G}=C \tilde{\mathbf{M}}^{-1} \tilde{\mathbf{N}} \mathbf{J}$, where $C \in R^{p \times n}, \mathbf{J} \in \mathcal{R H}_{\infty}^{p \times p}$ is diagonal and nonsingular on the $j \omega$-axis, $\tilde{\mathbf{M}} \in \mathcal{R} \mathcal{H}_{\infty}^{n \times n}, \tilde{\mathbf{N}} \in \mathcal{R} \mathcal{H}_{\infty}^{n \times p}$. This is the type

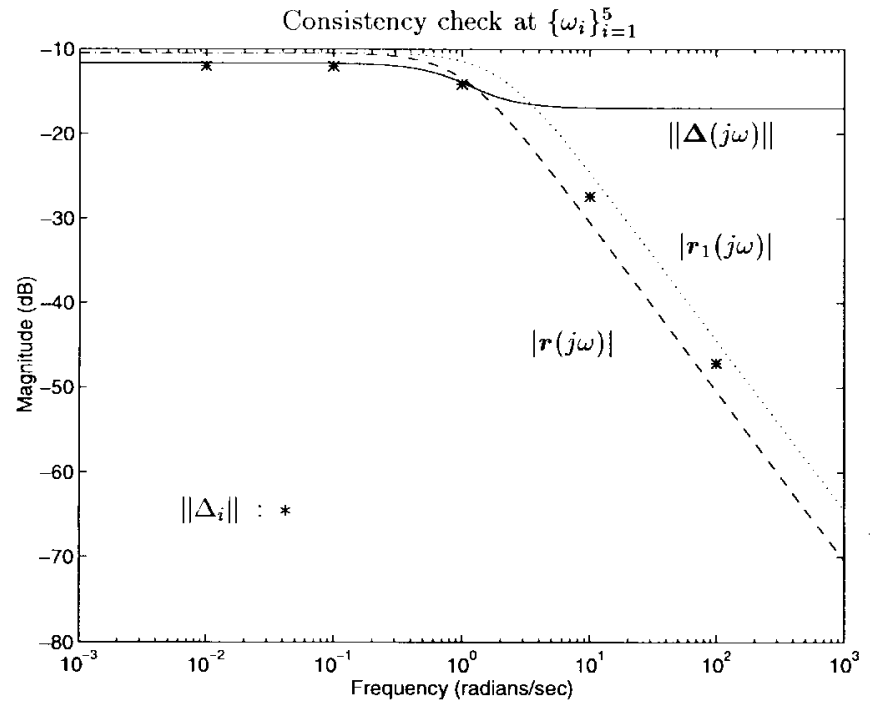

Fig. 3. Consistency check for the noise-free MIMO example.

of factorization proposed for LFSS in [5]. In this case, $n$ is the number of modes and the factor perturbations are mainly induced by variations in the modal parameters of the system. From [5], the input and output matrices are $\mathbf{J}=\gamma J_{2} \mathbf{T}_{a}$ and $C=d_{\max }^{-1} C_{1} J_{1}$, where $\mathbf{T}_{a}$ is a diagonal transfer matrix in $\mathcal{R} \mathcal{H}_{\infty}^{p \times p}$ modeling actuator dynamics.

As before, A1) is assumed so that the perturbed plant is stable for all $\Delta \in \mathcal{D}_{r_{\tilde{r}}}$. Fix a measurement frequency $\omega$ and let $J:=\mathbf{J}(j \omega), \tilde{M}:=\tilde{\mathbf{M}}(j \omega), \tilde{N}:=\tilde{\mathbf{N}}(j \omega), \Delta_{M}:=\boldsymbol{\Delta}_{M}(j \omega)$, $\Delta_{N}:=\boldsymbol{\Delta}_{N}(j \omega), \Delta:=\boldsymbol{\Delta}(j \omega), r:=\mathbf{r}(j \omega)$ and $\tilde{\Delta}:=r^{-1} \Delta$. The following assumption is made.

A2) The datum $\Phi \in C^{p \times p}$ is invertible.

This property should be generic in practice. The consistency equation at frequency $\omega$ is

$$
\Phi-C\left(\tilde{M}+\Delta_{M}\right)^{-1}\left(\tilde{N}+\Delta_{N}\right) J=0 .
$$

The model/data consistency problem that we want to solve is the following.

Problem 3: Given noise-free, invertible, open-loop frequency-response data $\left\{\Phi_{i}\right\}_{i=1}^{N} \subset C^{p \times p}$ at the distinct frequencies $\omega_{1}, \cdots, \omega_{N}$, could they have been produced by at least one model in $\mathcal{P}$ ? Or, in other words, does there exist $\boldsymbol{\Delta} \in \mathcal{D}_{r}$ such that $\mathbf{G}_{p}\left(j \omega_{i}\right)=\Phi_{i}, i=1, \cdots, N$ ?

Note that this problem is not as easily solved as the previous one because in general $C$ is not square. Let $\hat{\Phi}:=\Phi J^{-1}$. For convenience, let us postmultiply both sides of (9) by $J^{-1}$ and rewrite it using LFT notation

$$
\hat{\Phi}-\mathcal{F}_{U}(P, \tilde{\Delta})=0
$$

where

$$
\begin{aligned}
P & :=\left[\begin{array}{ll}
P_{11} & P_{12} \\
P_{21} & P_{22}
\end{array}\right] \\
P_{11} & :=\left[\begin{array}{c}
0 \\
r \tilde{M}^{-1}
\end{array}\right], \quad P_{12}:=\left[\begin{array}{c}
r I \\
r \tilde{M}^{-1} \tilde{N}
\end{array}\right] \\
P_{21} & :=C \tilde{M}^{-1}, \quad P_{22}:=C \tilde{M}^{-1} \tilde{N} .
\end{aligned}
$$




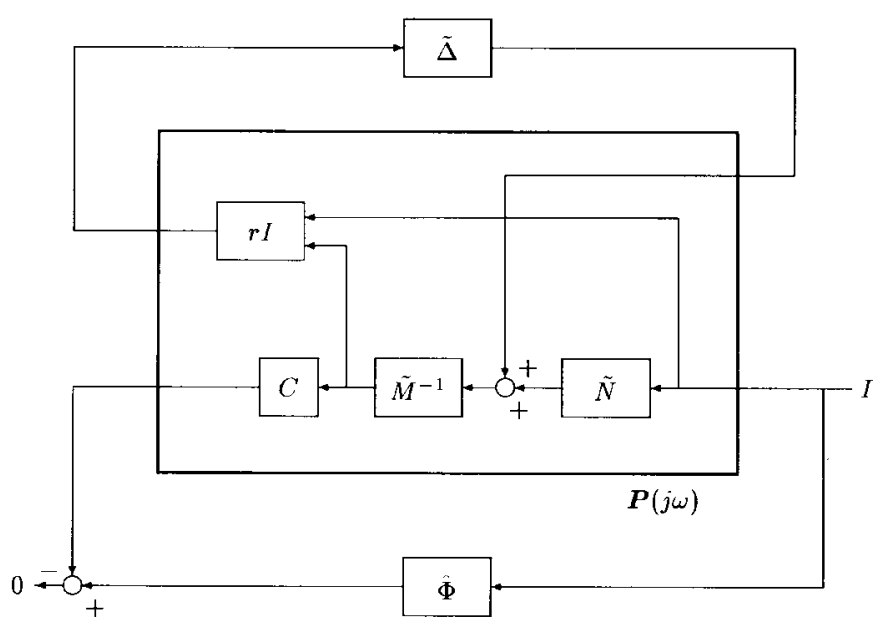

Fig. 4. Block diagram of consistency equation for noise-free MIMO flexible system.

This consistency equation is illustrated in Fig. 4. We make three other assumptions.

A3) $n \geq p$;

A4) $C$ has full row rank;

A5) $\hat{\Phi}-P_{22}$ is nonsingular.

Assumption A3) says that there are more modes in the model than there are outputs (and inputs). Assumptions A3) and A4) hold for most LFSS or experimental testbeds and are not really restrictive. Some motivation for these assumptions is provided by the following observation. Referring to Fig. 4, we can see that a necessary condition for consistency is that the columns of the $p \times p$ matrix $\left(\hat{\Phi}-P_{22}\right)$ lie in $\operatorname{Ra}\left\{P_{21}\right\}$, where $\hat{\Phi}$ is the scaled datum and $P_{21}$ is $p \times n$. But we have to assume that $\hat{\Phi}-P_{22}$ is nonsingular [Assumption A5)] for Lemma 4 to hold true, so it follows that $P_{21}$ must have full row rank $p$. This is turn implies that we must have $n \geq p$.

Let $P_{i}:=\mathbf{P}\left(j \omega_{i}\right), J_{i}:=\mathbf{J}\left(j \omega_{i}\right)$, and $\hat{\Phi}_{i}:=\Phi_{i} J_{i}^{-1}$. Then the main result for the square MIMO model/data consistency problem with noise-free invertible measurements and special factorization for flexible systems is the following.

Theorem 3: Problem 3 has a positive answer iff $\sigma_{p}\left[\mathcal{F}_{L}\right.$ $\left.\left(P_{i}, \hat{\Phi}_{i}^{-1}\right)\right]^{-1}<1$ for $i=1, \cdots, N$.

The proof is given in Appendix B. The problem is first decomposed into $N$ constant matrix optimization problems, which are solved using the Schmidt-Mirsky theorem [23, Th. 4.18]. Then the boundary interpolation theorem (Theorem 6) is invoked to complete the proof.

\section{B. Noisy SISO Case}

The model/data consistency problem will be studied for standard and special LCF's of SISO plants with noisy openloop experimental data. Suppose we are given $N$ nonzero noisy scalar frequency-response measurements $\left\{\phi_{i}\right\}_{i=1}^{N} \subset C$ corresponding to the distinct frequencies $\omega_{1}, \cdots, \omega_{N}$. For more generality, we consider a stable nominal LCF of the plant where the factor $\tilde{\mathbf{M}}$ has dimensions $n \times n$ and the factor $\tilde{\mathbf{N}}$ has dimensions $n \times 1$, together with an output transfer matrix $\mathbf{C}$ of dimension $1 \times n$. Any input scaling factor or actuator dynamics is lumped into $\mathbf{C}$, so referring to [5], for an $n$ mode SISO LFSS, we have $\mathbf{C}=d_{\max }^{-1} \gamma J_{2} \mathbf{T}_{a} C_{1} J_{1}$, where $\mathbf{T}_{a}$, $J_{2}, d_{\max }$, and $\gamma$ are scalars. Thus the nominal plant transfer function $\mathbf{g}$ is written as

$$
\mathbf{g}=\mathbf{C} \tilde{\mathbf{M}}^{-1} \tilde{\mathbf{N}} \text {. }
$$

A unit $\mathbf{r}(s)$ in $\mathcal{R} \mathcal{H}_{\infty}$ bounding the factor uncertainty is also part of the model as before.

Define the normalized perturbations $\tilde{\boldsymbol{\Delta}}_{N}:=\mathrm{r}^{-1} \boldsymbol{\Delta}_{N}$, $\tilde{\boldsymbol{\Delta}}_{M}:=\mathrm{r}^{-1} \boldsymbol{\Delta}_{M}$, and the normalized uncertainty matrix

$$
\tilde{\boldsymbol{\Delta}}:=\left[\begin{array}{ll}
\tilde{\boldsymbol{\Delta}}_{N} & -\tilde{\boldsymbol{\Delta}}_{M}
\end{array}\right]=\mathrm{r}^{-1} \boldsymbol{\Delta} .
$$

The factor uncertainty set $\mathcal{D}_{r}$ and the family of perturbed plants $\mathcal{P}$ are as defined in (2) and (3), respectively. The scalar noise set $\mathcal{W}$ was defined in (5). The noisy open-loop SISO consistency problem can be stated as follows.

Problem 4: Given nonzero noisy open-loop frequencyresponse data $\left\{\phi_{i}\right\}_{i=1}^{N} \subset C$ at $\omega_{1}, \cdots, \omega_{N}$, could they have been produced by at least one model in $\mathcal{P}$ and $N$ noises in $\mathcal{W}$ ? Or, in other words, do there exist $\boldsymbol{\Delta} \in \mathcal{D}_{r}$ and complex noises $\left\{w_{a i}\right\}_{i=1}^{N}$ in $\mathcal{W}$ such that $\mathrm{g}_{p}\left(j \omega_{i}\right)+w_{a i}=\phi_{i}, i=1, \cdots, N$ ?

Fix a measurement frequency $\omega$ and let $\phi$ be the complex measurement at that frequency. For the nominal LCF, let $C:=\mathbf{C}(j \omega), \tilde{\Delta}_{M}:=\tilde{\boldsymbol{\Delta}}_{M}(j \omega)$, and $\tilde{\Delta}_{N}:=\tilde{\boldsymbol{\Delta}}_{N}(j \omega)$. The normalized additive complex noise at frequency $\omega$ is $\tilde{w}_{a}:=L_{a}^{-1} w_{a}$ where $w_{a} \in \mathcal{W}$. The generalized consistency equation at frequency $\omega$ is

$$
\phi-C\left(\tilde{M}+r \tilde{\Delta}_{M}\right)^{-1}\left(\tilde{N}+r \tilde{\Delta}_{N}\right)-L_{a} \tilde{w}_{a}=0 .
$$

Using LFT notation, (13) becomes

$$
\phi-\mathcal{F}_{U}\left[\mathbf{V}(j \omega), \tilde{\Delta}_{s}\right]=0
$$

where

$$
\tilde{\Delta}_{s}:=\left[\begin{array}{cc}
\tilde{\Delta} & 0 \\
0 & \tilde{w}_{a}
\end{array}\right]
$$

and

$$
\begin{array}{rlrl}
\mathbf{V} & :=\left[\begin{array}{ll}
\mathbf{V}_{11} & \mathbf{V}_{12} \\
\mathbf{V}_{21} & \mathbf{V}_{22}
\end{array}\right] \\
\mathbf{V}_{11} & :=\left[\begin{array}{cc}
0 & 0 \\
\tilde{r}^{-1} & 0 \\
0 & 0
\end{array}\right], & \mathbf{V}_{12}:=\left[\begin{array}{c}
\mathbf{r} \\
\mathbf{r} \tilde{\mathbf{M}}^{-1} \tilde{\mathbf{N}} \\
1
\end{array}\right] \\
\mathbf{V}_{21} & :=\left[\begin{array}{ll}
\tilde{\mathbf{C M}}^{-1} & L_{a}
\end{array}\right], & \mathbf{V}_{22}:=\mathbf{C M}^{-1} \tilde{\mathbf{N}} .
\end{array}
$$

Note that $V, V_{i j}$ are defined in the usual way at frequency $\omega$. The block diagram of Fig. 5 represents a general model/data consistency equation at frequency $\omega$ that can be specialized to the two different consistency problems considered. Referring to the block diagram, these configurations are: 1) Scalar factorization $(n=1)$, i.e., $\tilde{\mathbf{M}} \in \mathcal{R} \mathcal{H}_{\infty}^{1 \times 1}, \tilde{\mathbf{N}} \in \mathcal{R} \mathcal{H}_{\infty}^{1 \times 1}, \mathbf{C}=1$ and 2) Special factorization for LFSS, i.e., $\tilde{\mathbf{M}} \in \mathcal{R} \mathcal{H}_{\infty}^{n \times n}$, $\tilde{\mathbf{N}} \in \mathcal{R} \mathcal{H}_{\infty}^{n \times 1}, \mathbf{C} \in \mathcal{R} \mathcal{H}_{\infty}^{1 \times n}$. The following assumptions are made.

A6) $\phi \neq 0$ (nonzero complex measurement);

A7) $\left(\phi-V_{22}\right) \neq 0$. 


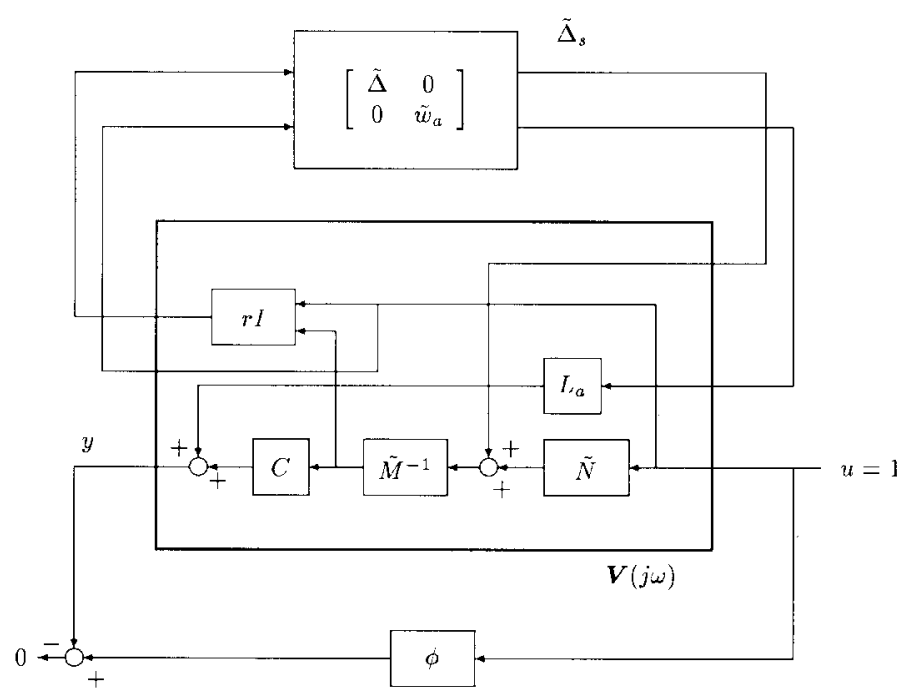

Fig. 5. Block diagram of consistency equation for noisy open-loop SISO flexible system at frequency $\omega$.

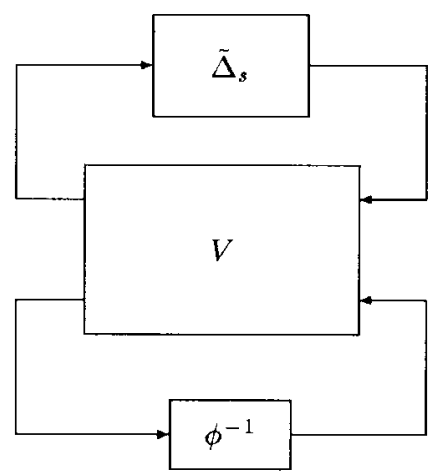

Fig. 6. Block diagram of equivalent feedback problem.

These assumptions are required for invertibility of the complex numbers $\phi$ and $\left(\phi-V_{22}\right)$ in the proofs, and should hold generically in practice. The general consistency problem at frequency $\omega$ can be stated as follows.

Problem 5: Given a nonzero noisy frequency-response datum $\phi$ at frequency $\omega$, do there exist $\tilde{\Delta} \in \mathcal{B} C^{n \times(n+1)}$ and a complex noise $w_{a} \in \mathcal{W}$ such that $\phi-\mathcal{F}_{U}\left(V, \tilde{\Delta}_{s}\right)=0$ ?

The key idea for solving this problem is to write a consistency equation equivalent to (14), but that has a feedback interpretation as in Fig. 6

$$
1-\phi^{-1} \mathcal{F}_{U}\left(V, \tilde{\Delta}_{s}\right)=0 .
$$

Then consistency becomes analogous to noninvertibility (or instability) of a feedback system at frequency $\omega$, and tools developed for stability analysis of linear systems, such as the structured singular value $\mu$, can be used to derive consistency results. Note that this approach is not new, since it was previously used in, e.g., [22] and [19]. Based on this idea, the following lemma forms a basis for the solution of the consistency problem.

Lemma 1: For a frequency $\omega \in R_{+}$, we are given a nonzero complex measurement $\phi$. Then for $\Delta \in C^{(n+1) \times(n+2)}$ with
$I-V_{11} \Delta$ nonsingular, $\phi-\mathcal{F}_{U}(V, \Delta)=0$ iff $I-\mathcal{F}_{L}\left(V, \phi^{-1}\right) \Delta$ is singular.

A proof for this lemma is given in Appendix C. Define the structured perturbation set

$$
\Gamma:=\left\{\tilde{\Delta}_{s}=\left[\begin{array}{cc}
\tilde{\Delta} & 0 \\
0 & \tilde{w}_{a}
\end{array}\right]: \tilde{\Delta} \in C^{n \times(n+1)}, \tilde{w}_{a} \in C\right\} .
$$

This set will be useful in deriving the consistency results for the general factorization. Recall that the structured singular value $\mu_{\Gamma}: C^{(n+2) \times(n+1)} \rightarrow R_{+}$corresponding to the structured perturbations in $\Gamma$ is defined as follows [9]:

$$
\mu_{\Gamma}(M):=\left[\min \left\{\left\|\tilde{\Delta}_{s}\right\|: \tilde{\Delta}_{s} \in \Gamma, \operatorname{det}\left(I-M \tilde{\Delta}_{s}\right)=0\right\}\right]^{-1}
$$

unless no $\tilde{\Delta}_{s} \in \Gamma$ makes $I-M \tilde{\Delta}_{s}$ singular, in which case $\mu_{\Gamma}(M):=0$.

The main result of this section, proven in Appendix C, can now be stated. It gives a necessary and sufficient condition for a positive answer to Problem 4, the consistency problem for noisy open-loop measurements on SISO plants.

Theorem 4: Problem 4 has a positive answer, i.e., there exist $\mathbf{g}_{p} \in \mathcal{P}$ and noises $w_{a i} \in \mathcal{W}, i=1, \cdots, N$, that could have produced the $\phi_{i}$ 's, iff $\mu_{\Gamma}\left\{\mathcal{F}_{L}\left[\mathbf{V}\left(j \omega_{i}\right), \phi_{i}^{-1}\right]\right\}>1$ for $i=1, \cdots, N$.

Suppose that for a given set of frequency-response measurements, the $\mu$ test reveals that the LCF and noise models are inconsistent because at one measurement frequency $\omega_{j}$, $\mu_{\Gamma}\left\{\mathcal{F}_{L}\left[\mathbf{V}\left(j \omega_{j}\right), \phi_{j}^{-1}\right]\right\}$ is less than, but close to one. Then it may be possible to increase $\left|r_{j}\right|$ slightly to get consistency as Proposition 1 suggests, which follows from the fact that if $\left|r_{j}^{\prime}\right|>\left|r_{j}\right|$, then $\left\{\Delta:\|\Delta\|<\left|r_{j}\right|\right\} \subset\left\{\Delta:\|\Delta\|<\left|r_{j}^{\prime}\right|\right\}$.

Proposition 1: $\mu_{\Gamma}\left\{\mathcal{F}_{L}\left[\mathbf{V}\left(j \omega_{j}\right), \phi_{j}^{-1}\right]\right\}$ is a nondecreasing function of $\left|r_{j}\right|$ on $\left[0, \underline{\sigma}\left(\tilde{M}_{j}\right)\right)$.

Numerical Example: Experimental frequency-response data obtained on a planar two-link flexible robot [18] are used for a numerical example illustrating the result. The plant has two motor voltage inputs at the joints and two outputs, namely the tip position of the first link and the angle of the second joint. The measurements were taken in the zero-angle configuration. Here we want to test a coprime factor model of the SISO plant from the motor voltage of the first joint to the tip position of the first link.

A factorization was derived from the nominal model of the flexible robot given in [18], and a bound $\mathbf{r}$ on the factor uncertainty was obtained using the method proposed in [5] for factorizations of LFSS's. Note that Assumption A1) holds. The noise level in the data was estimated to be roughly $L_{a}=0.06$. The measurements were taken at 211 distinct frequencies ranging from 1 to $64 \mathrm{rad} / \mathrm{s}$. Here is the nominal tenth-order model $\mathbf{g}=C \tilde{\mathbf{M}}^{-1} \tilde{\mathbf{N}}$ composed of five flexible modes

$$
\mathbf{g}(s)=C \tilde{\mathbf{M}}^{-1} \tilde{\mathbf{N}}
$$




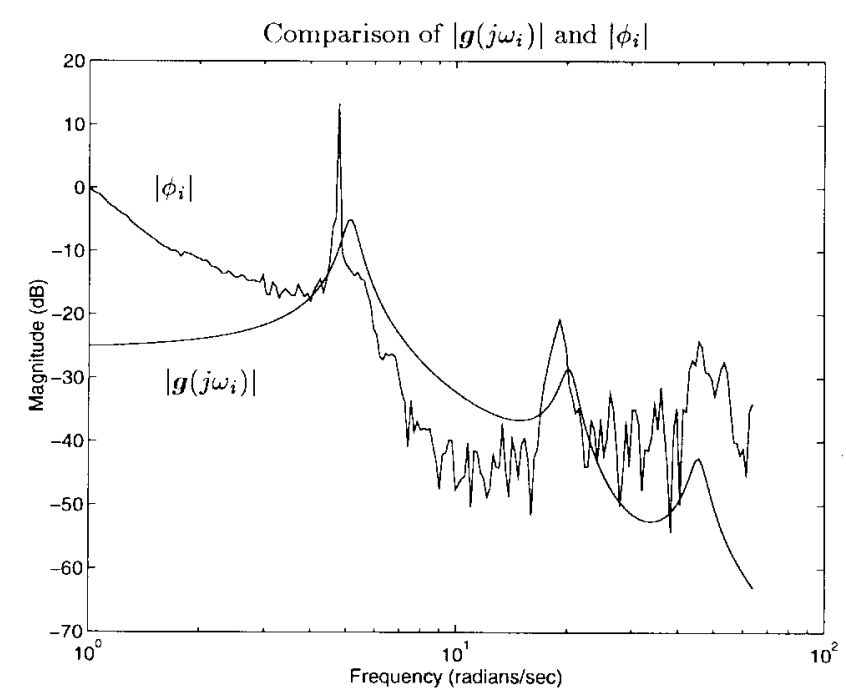

Fig. 7. Fit between the magnitudes of the measured frequency response and the nominal model.

where

$$
\begin{aligned}
& \tilde{\mathbf{M}}(s)= \frac{1}{s^{2}+41.2 s+40.2} \\
& \cdot \operatorname{diag}\left\{4.0 s^{2}+4.1 s+105.0,4.0 s^{2}+36.1 s+8228.6\right. \\
& 4.0 s^{2}+16.1 s+1634.0,4.0 s^{2}+90.8 s \\
&\left.+20116,4.0 s^{2}+22.8 s+1310.3\right\}
\end{aligned}
$$$$
\tilde{\mathbf{N}}(s)=\frac{1}{s^{2}+41.2 s+40.2}\left[\begin{array}{c}
1800 \\
-1800 \\
-1800 \\
1800 \\
1800
\end{array}\right]
$$$$
C=10^{-3}\left[\begin{array}{lllll}
-3.30 & 3.40 & -3.39 & 0.00 & -0.01
\end{array}\right] .
$$

Notice that rigid-body modes were not included in order to keep the nominal model stable, although the data in Fig. 7 show that there seem to be two poles at $s=0$, as one would expect. Nonetheless, we will check if the data are consistent with this model and the noise level $L_{a}$. The weighting function bounding the factor uncertainty is $\mathbf{r}(s)=\frac{\tilde{5} \times 10^{-4} s+.75}{s+1}$.

The structured singular value $\mu_{\Gamma}\left\{\mathcal{F}_{L}\left[\mathbf{V}\left(j \omega_{i}\right), \phi_{i}^{-1}\right]\right\}$ is plotted in Fig. 8. Because the structured perturbation $\tilde{\Delta}_{s}$ has only one scalar block and one full block, $\mu_{\Gamma}$ can be computed to any desired accuracy [1]. It is seen to be smaller than one at many measurement frequencies. Hence, it can be concluded that the family of plants $\mathcal{P}$ and the noise set $\mathcal{W}$ are not consistent with the data using the selected $\mathbf{r}$. One would have to reshape the bound $\mathbf{r}$ and perhaps even adopt a new nominal coprime factorization to get consistency. Note that once the LFT's were computed, the $\mu$-test only took a few seconds on a Sun SPARCstation ${ }^{\mathrm{TM}} 10$.

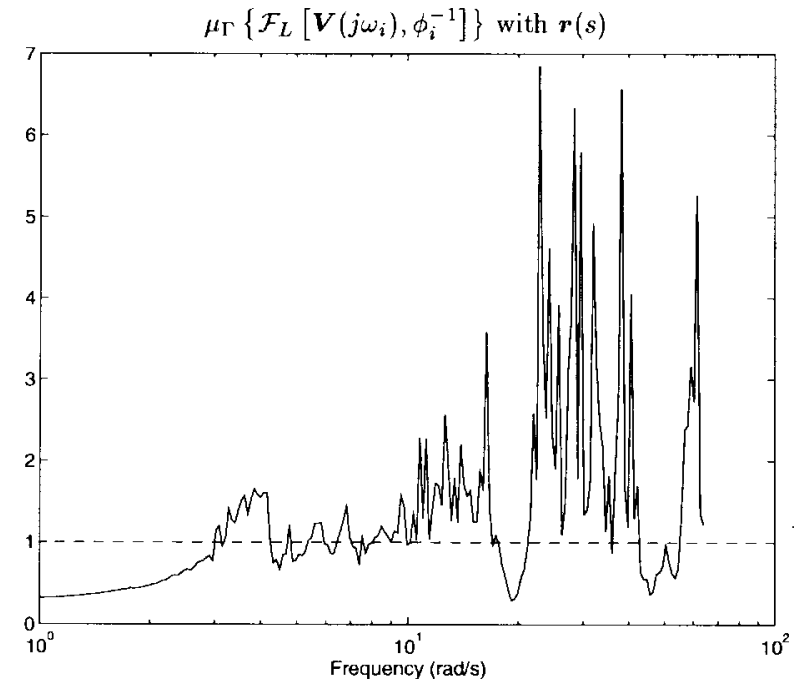

Fig. 8. Structured singular value test for consistency with $\mathbf{r}$.

\section{CONCLUSION}

The general model/data consistency problem was formulated and specialized to perturbed coprime factor models and frequency-response data. The first problem considered, the open-loop noise-free MIMO case, led to a boundary interpolation theorem in $\mathcal{R H}_{\infty}$. This theorem was used for the other cases as well and allowed us to focus on consistency at one measurement frequency at a time. This simplifies the problem because then the consistency equations involve constant complex matrices only. Hence the tests for consistency boil down to showing that there exist constant factor perturbations of norm less than one such that the complex consistency equations hold at each measurement frequency. These tests involve singular value or structured singular value computations on LFT's constructed with the complex frequency-response data and the a priori information of the nominal coprime factorization and the factor uncertainty and noise bounds. In the open-loop case it was shown that the bound $|\mathrm{r}(j \omega)|$ on the factor uncertainty can be modified such that consistency is obtained for all measurements. It is now clear how $\mathbf{r}$ can be constructed and improved as new experimental data become available. This allows the control system designer to reduce the uncertainty about the size of the plant's uncertainty.

\section{APPENDIX A \\ PROOF OF THEOREM 2}

A few results must be introduced before the Proof of Theorem 2 can be presented.

The interpolation problem at hand can be scaled as follows: find a function $\Psi \in \mathcal{R} \mathcal{H}_{\infty}$ interpolating the product $\mathrm{r}^{-1}\left(j \omega_{i}\right) \Delta_{i}$ at $s=j \omega_{i}, i=1, \cdots, N$ and such that $\|\Psi\|_{\infty}<$ 1. The interpolation problem in $\mathcal{R} \mathcal{H}_{\infty}$ of the right half-plane is then transformed into an interpolation problem in $\mathcal{R H}_{\infty}(D)$ by using the one-to-one scalar bilinear transformation $s \longmapsto z$ defined by $z=(1-s) /(1+s)$ which maps the closed right half-plane onto the closed unit disk $\bar{D}$. Thus the boundary interpolation problem can be stated as follows. 
Problem 6: Given a set of distinct points $\left\{e^{j \theta_{i}}\right\}_{i=1}^{r}$ on the unit circle $\partial D$ and a set $\left\{\tilde{\Psi}_{i}\right\}_{i=1}^{r}$ in $C^{m \times n}$ satisfying $\left\|\Psi_{i}\right\|<1$ for $i=1, \cdots, N, \tilde{\Psi}_{l}=\left(\tilde{\Psi}_{j}^{*}\right)^{T}$ for $\theta_{l}=-\theta_{j}$, and $\widetilde{\Psi}_{k} \in R^{m \times n}$ for $\theta_{k}=0, \pm \pi$, does there exist a function $\tilde{\boldsymbol{\Psi}} \in \mathcal{R} \mathcal{H}_{\infty}(D)$, $\|\tilde{\boldsymbol{\Psi}}\|_{\infty}<1$ such that $\tilde{\boldsymbol{\Psi}}\left(e^{j \theta_{i}}\right)=\tilde{\Psi}_{i} i=1, \cdots, r$ ?

To solve Problem 6, a classical result on interpolation in $D$ from Fedčina [10] (see also [8]) is needed. A modified version of it in [24, Th. 71] leads to a corollary that gives a necessary and sufficient condition for the following matrix Nevanlinna-Pick problem to have a solution.

Problem 7: Given a set of distinct points $\left\{z_{i}\right\}_{i=1}^{r}$ in $D$ and a set $\left\{\Theta_{i}\right\}_{i=1}^{r}$ in $C^{p \times p}$ satisfying $\left\|\Theta_{i}\right\|<1, i=1, \cdots, r$, $\Theta_{l}=\left(\Theta_{j}^{*}\right)^{T}$ for $z_{l}=z_{j}^{*}$, and $\Theta_{k} \in R^{p \times p}$ for $z_{k} \in(-1,1)$, does there exist a matrix $\Theta \in \mathcal{B R H}_{\infty}(D)^{p \times p}$ such that $\Theta\left(z_{i}\right)=\Theta_{i}, i=1, \cdots, r$ ?

The result derived from [24, Th. 71] goes as follows.

Theorem 5: Problem 7 has a solution iff the block-Pick matrix $P$ is positive definite, where

$$
P:=\left[\begin{array}{ccc}
\frac{I-\Theta_{1}^{*} \Theta_{1}}{1-z_{1}^{*} z_{1}} & \cdots & \frac{I-\Theta_{1}^{*} \Theta_{r}}{1-z_{1}^{*} z_{r}} \\
\vdots & \ddots & \vdots \\
\frac{I-\Theta_{r}^{*} \Theta_{1}}{1-z_{r}^{*} z_{1}} & \cdots & \frac{I-\Theta_{r}^{*} \Theta_{r}}{1-z_{r}^{*} z_{r}}
\end{array}\right] .
$$

Proof: We see in [24, Th. 71] that there exists a function $\Theta \in \mathcal{B C H}_{\infty}(D)^{p \times p}$ interpolating the matrices $\Theta_{i}$ as given in Problem 7 iff $P$ is positive definite. This result is equivalent to the theorem statement for Problem 7 modified so that $\Theta$ is allowed to be in $\mathcal{B C H}_{\infty}(D)^{p \times p}$, the open unit ball of $p \times p$ complex-rational matrices in $\mathcal{H}_{\infty}(D)$. Based on this, we are ready to prove the two implications in the theorem statement.

Sufficiency: If $P$ is positive definite, then there exists $\boldsymbol{\Phi}$ in $\mathcal{B C H}_{\infty}(D)^{p \times p}$ such that $\boldsymbol{\Phi}\left(z_{i}\right)=\Theta_{i}, i=1, \cdots, N$. Let $\underline{\mathbf{\Phi}}(z)$ denote the complex-rational matrix-valued function $\boldsymbol{\Phi}(z)$ with its coefficients replaced by their complex conjugates. Define the function

$$
\Theta(z):=\frac{1}{2}[\Phi(z)+\underline{\Phi}(z)] .
$$

Note that $\Theta(z) \in \mathcal{B R H}_{\infty}(D)^{p \times p}$ and it interpolates the matrices $\Theta_{i}$.

Necessity: Suppose $P$ is not positive definite. Then by [24, Th. 71], there exists no function in $\mathcal{B C H}_{\infty}(D)^{p \times p}$ interpolating the $\Theta_{i}$ 's. It follows that there exists no function in $\mathcal{B R H}_{\infty}(D)^{p \times p}$ interpolating these same matrices as $\mathcal{B R H}_{\infty}(D)^{p \times p} \subset \mathcal{B C} \mathcal{H}_{\infty}(D)^{p \times p}$.

For interpolation points on the unit circle $\partial D$, it is not possible to use Theorem 5 directly. Consider the mapping $g_{\epsilon}: C \rightarrow C$ defined by $g_{\epsilon}(z):=z /(1+\epsilon), z \in C, \epsilon>0$.
Now define the open disk of radius $1+\epsilon$

$$
D_{1+\epsilon}:=\{z:|z|<1+\epsilon\}
$$

and consider the class $\mathcal{B R H}_{\infty}\left(D_{1+\epsilon}\right)^{p \times p}$ of square matrixvalued real-rational functions analytic and with norm less than one on $D_{1+\epsilon}$.

Lemma 2: For $\epsilon$ small enough, there exists a function in $\mathcal{B R} \mathcal{H}_{\infty}\left(D_{1+\epsilon}\right)^{p \times p}$ solving Problem 7 modified such that the interpolation points $\left\{z_{i}\right\}_{i=1}^{r}$ are on the unit circle.

The proof requires the following proposition from [13, $\mathrm{p}$. 367, ex. 2].

Proposition 2: Let $A \in C^{q \times q}, E \in C^{q \times q}$ be both Hermitian. Let $\lambda_{1} \leq \cdots \leq \lambda_{q}$ be the ordered eigenvalues of $A$ and $\hat{\lambda}_{1} \leq \cdots \leq \hat{\lambda}_{q}$ be the ordered eigenvalues of $A+E$. Then $\left|\lambda_{k}-\hat{\lambda}_{k}\right| \leq \rho(E), k=1, \cdots, q$.

Proof of Lemma 2: Apply $g_{\epsilon}$ to $z_{i} \in \partial D$ to get $\left\{\tilde{z}_{i}(\epsilon):=\right.$ $\left.z_{i} /(1+\epsilon)\right\}_{i=1}^{r}$. Construct the block-Pick matrix for Problem 7 but for the $\tilde{z}_{i}(\epsilon)$ 's in $D$ instead of the $z_{i}$ 's on the unit circle as shown in (24), at the bottom of the page. Note that $P$ is positive definite iff $\epsilon P$ is positive definite. Write $\epsilon P$ as

$$
\epsilon P=P_{a}+P_{e}
$$

where

$$
\begin{aligned}
P_{a}:=\operatorname{blockdiag}\left\{\frac{\left(I-\Theta_{1}^{*} \Theta_{1}\right)(1+\epsilon)^{2}}{2+\epsilon},\right. \\
\left.\cdots, \frac{\left(I-\Theta_{r}^{*} \Theta_{r}\right)(1+\epsilon)^{2}}{2+\epsilon}\right\}
\end{aligned}
$$

and $P_{e}:=\epsilon P-P_{a}$. Note that each nonzero (off-diagonal) block in $P_{e}$ has $\epsilon$ as a factor. Clearly $P_{a}$ is positive definite, thus $\lambda_{i}\left(P_{a}\right)>0, i=1, \cdots, r p$. Also, note that $P_{a}$ and $P_{e}$ are both Hermitian. Identifying $A$ with $P_{a}$ and $E$ with $P_{e}$ in Proposition 2, we get the following inequality:

$$
\left|\lambda_{k}-\hat{\lambda}_{k}\right| \leq \rho\left(P_{e}\right), \quad k=1, \cdots, r p .
$$

This says that since we can make the spectral radius $\rho\left(P_{e}\right)$ as small as we want by proper choice of $\epsilon$, we can make it smaller than $\lambda_{1}$ for some $\epsilon_{1}$, thereby causing $\epsilon_{1} P$ to be positive definite. Hence there exists $\Theta \in \mathcal{B R \mathcal { H }}_{\infty}(D)^{p \times p}$ solving Problem 7 for the set of interpolation points $\left\{\tilde{z}_{i}\left(\epsilon_{1}\right)\right\}_{i=1}^{r} \subset$ $D$. Now let $\boldsymbol{\Psi}:=\boldsymbol{\Theta} \circ g_{\epsilon_{1}}$, i.e., $\boldsymbol{\Psi}(z)=\boldsymbol{\Theta}\left(g_{\epsilon_{1}}(z)\right)=$ $\boldsymbol{\Theta}\left(z /\left(1+\epsilon_{1}\right)\right)$. Then $\boldsymbol{\Psi}: D_{1+\epsilon_{1}} \rightarrow C^{p \times p}$ is analytic, belongs to $\mathcal{B R H}_{\infty}\left(D_{1+\epsilon}\right)^{p \times p}$, and takes on the value $\Theta_{k}$ at $z_{k}$ for $k=1, \cdots, r$.

The idea of extending $D$ to $D_{1+\epsilon}$ and using the function $g_{\epsilon}$ comes from [14]. We now have all the tools to establish the following result on boundary interpolation which gives an answer to Problem 6. Note that $[2$, Ch. 21] is an excellent

$$
P=\left[\begin{array}{cccc}
\frac{\left(I-\Theta_{1}^{*} \Theta_{1}\right)(1+\epsilon)^{2}}{\epsilon(2+\epsilon)} & \frac{\left(I-\Theta_{1}^{*} \Theta_{2}\right)(1+\epsilon)^{2}}{(1+\epsilon)^{2}-z_{2} z_{1}^{*}} & \cdots & \frac{\left(I-\Theta_{1}^{*} \Theta_{r}\right)(1+\epsilon)^{2}}{(1+\epsilon)^{2}-z_{r} z_{1}^{*}} \\
\frac{\left(I-\Theta_{2}^{*} \Theta_{1}\right)(1+\epsilon)^{2}}{(1+\epsilon)^{2}-z_{1} z_{2}^{*}} & \ddots & \vdots \\
\vdots & & & \vdots \\
\frac{\left(I-\Theta_{r}^{*} \Theta_{1}\right)(1+\epsilon)^{2}}{(1+\epsilon)^{2}-z_{1} z_{r}^{*}} & \cdots & \frac{\left(I-\Theta_{r}^{*} \Theta_{r}\right)(1+\epsilon)^{2}}{\epsilon(2+\epsilon)}
\end{array}\right]
$$


source for problems related to boundary Nevanlinna-Pick interpolation.

Theorem 6: Given a set of distinct points $\left\{e^{j \theta_{i}}\right\}_{i=1}^{r}$ on $\partial D$ and a set $\left\{\tilde{\Psi}_{i}\right\}_{i=1}^{r}$ in $C^{m \times n}$ satisfying $\widetilde{\Psi}_{l}=\left(\tilde{\Psi}_{j}^{*}\right)^{T}$ for $\theta_{l}=$ $-\theta_{j}$ and $\tilde{\Psi}_{k} \in R^{m \times n}$ for $\theta_{k}=0, \pm \pi$, there exists a function $\tilde{\boldsymbol{\Psi}} \in \mathcal{B R}_{\mathcal{H}}(D)^{m \times n}$ satisfying $\tilde{\boldsymbol{\Psi}}\left(e^{j \theta_{i}}\right)=\tilde{\Psi}_{i}, i=1, \cdots, r$ iff $\left\|\tilde{\Psi}_{i}\right\|<1$ for $i=1, \cdots, r$.

Proof (Necessity): The proof follows immediately from the bound on the norm of $\tilde{\boldsymbol{\Psi}}$.

Sufficiency: Let $q=\max \{m, n\}$. Append zeros to the complex matrices $\left\{\widetilde{\Psi}_{k}\right\}_{k=1}^{r}$ to make them square

$$
\begin{aligned}
& \Gamma_{k}:=\left[\begin{array}{c}
\tilde{\Psi}_{k} \\
0
\end{array}\right] \quad \text { if } n=q, \quad k=1 \cdots, r, \\
& \Gamma_{k}:=\left[\tilde{\Psi}_{k} 0\right] \quad \text { if } m=q, \quad k=1, \cdots, r .
\end{aligned}
$$

Note that $\left\|\Gamma_{k}\right\|=\left\|\tilde{\Psi}_{k}\right\|<1, k=1, \cdots, r$. By Lemma 2, there exists $\boldsymbol{\Gamma} \in \mathcal{B} \mathcal{R} \mathcal{H}_{\infty}(D)^{q \times q}$ taking on the value $\Gamma_{k}$ at the boundary point $z_{k}=e^{j \theta_{k}}, k=1, \cdots, r$. Extract the $m \times n$ matrix $\tilde{\boldsymbol{\Psi}}(z)$ from the $q \times q$ matrix $\boldsymbol{\Gamma}(z)$ (the first $m$ rows and $n$ columns). Then, $\|\tilde{\boldsymbol{\Psi}}\|_{\infty} \leq\|\boldsymbol{\Gamma}\|_{\infty}<1$, and $\tilde{\boldsymbol{\Psi}}$ takes on the value $\tilde{\Psi}_{k}$ at the boundary point $z_{k}$, for $k=1, \cdots, r$.

With Theorem 6 in hand, proving our main result (Theorem 2) on consistency of the perturbed CF model with the noise-free experimental frequency-response data becomes a simple matter. Given a nominal coprime factor model $\mathbf{G}=\tilde{\mathbf{M}}^{-1} \tilde{\mathbf{N}}$, an uncertainty set $\mathcal{D}_{r}$ and a set of frequencyresponse measurements $\left\{\Phi_{i}\right\}_{i=1}^{N}$, it suffices to compute the minimum-norm complex matrices $\Delta_{i}$ for $i=1, \cdots, N$ and to check that $\| \Delta_{i}||<\left|\mathbf{r}\left(j \omega_{i}\right)\right|, \forall i=1, \cdots, N$. If this inequality does not hold for some $j \in\{1, \cdots, N\}$, then no perturbation of the coprime factors in $\mathcal{D}_{r}$ could have produced the data. This is summarized in the following Proof of Theorem 2.

Proof of Theorem 2: Scale the minimum-norm $\Delta_{i}$ with $\mathbf{r}$ to get $\widetilde{\Delta}_{i}:=\left|\mathbf{r}\left(j \omega_{i}\right)\right|^{-1} \Delta_{i}$ and compute $z_{i}=\frac{1-j \omega_{i}}{1+j \omega_{i}}$ for $i=1, \cdots, N$. Then apply Theorem 6 to show that there exists a function in $\mathcal{B R} \mathcal{H}_{\infty}(D)$ that interpolates the $\tilde{\Delta}_{i}$ 's at the $z_{i}$ 's iff $\left\|\tilde{\Delta}_{i}\right\|<1$ for all $i=1, \cdots, N$. Use the bilinear transformation on this function and unscale to get the factor perturbation $\boldsymbol{\Delta} \in \mathcal{D}_{r}$. Finally, notice that the corresponding $\mathbf{G}_{p}$ is stable from Assumption A1).

\section{APPENDIX B \\ PROOF OF THEOREM 3}

In order to establish Theorem 3, we need to study consistency at one frequency. The consistency problem at frequency $\omega$ is stated as follows.

Problem 8: Given a noise-free invertible frequencyresponse datum $\Phi \in C^{p \times p}$ at frequency $\omega$, does there exist $\tilde{\Delta} \in \mathcal{B} C^{n \times(n+p)}$ such that $C\left(\tilde{M}+\Delta_{M}\right)^{-1}\left(\tilde{N}+\Delta_{N}\right) J=\Phi$, i.e., $\hat{\Phi}-\mathcal{F}_{U}(P, \tilde{\Delta})=0$ ?

Let $P_{12}^{\dagger}$ be the Moore-Penrose left-inverse of the full column-rank matrix $P_{12}$ given by $P_{12}^{\dagger}:=\left(P_{12}^{*} P_{12}\right)^{-1} P_{12}^{*}$. It is easy to show that the left-nullspace $\mathcal{N}_{L}\left\{P_{12}\right\}=$ row span $\{[\tilde{N}-\tilde{M}]\}$, an $n$-dimensional subspace of $C^{p+n}$. Notice that $\left[\begin{array}{cc}P_{12}^{\dagger} & \tilde{N}_{N}\end{array}\right]$ is invertible and recall that subspaces are said to be independent if their intersection is $\{0\}$. The first lemma, proven in [4], is of a rather technical nature and is used in the proof of Lemma 4 which gives equivalent conditions for consistency at frequency $\omega$.

Lemma 3: Let $\mathcal{Y}:=\left\{\right.$ row span $\left(U P_{12}^{\dagger}+Q[\tilde{N}-\tilde{M}]\right): U$ $\in C^{p \times p}$ invertible, $\left.Q \in C^{p \times n}\right\}$. Then $\mathcal{Y}=\{p$-dimensional subspaces of $C^{p+n}$ that are independent of $\left.\mathcal{N}_{L}\left\{P_{12}\right\}\right\}$.

The following lemma gives equivalent conditions for consistency at frequency $\omega$.

Lemma 4: For $\omega \in R_{+}$, we are given the noise-free, invertible frequency-response measurement $\Phi \in C^{p \times p}$. Assume $\hat{\Phi}-P_{22}$ is invertible. Then for $\tilde{\Delta} \in \mathcal{B} C^{n \times(p+n)}$, the following consistency conditions are equivalent.

1) $\hat{\Phi}-\mathcal{F}_{U}(P, \tilde{\Delta})=0$.

2) $\left(P_{12}^{\dagger}-Q[\tilde{N}-\tilde{M}]\right)\left[I-\mathcal{F}_{L}\left(P, \hat{\Phi}^{-1}\right) \tilde{\Delta}\right]=0$ for some $Q \in C^{p \times n}$.

3) $\operatorname{rank}\left[I-\mathcal{F}_{L}\left(P, \hat{\Phi}^{-1}\right) \tilde{\Delta}\right] \leq n$.

Proof: First note that $\tilde{\Delta} \in \mathcal{B} C^{n \times(p+n)}$ implies that $I-P_{11} \tilde{\Delta}$ is nonsingular by Assumption A1).

$1) \Leftrightarrow 2$ ): This follows after simple algebraic manipulations on the consistency equation in 1); see [4] for details.

$3) \Rightarrow 2)$ : Suppose that 3$)$ holds. Then $\operatorname{dim}\left(\mathcal{N}_{L}\{I-\right.$ $\left.\left.\mathcal{F}_{L}\left(P, \hat{\Phi}^{-1}\right) \tilde{\Delta}\right\}\right) \geq p$. We now show that $\mathcal{N}_{L}\left\{P_{12}\right\}$ and $\mathcal{N}_{L}\left\{I-\mathcal{F}_{L}\left(P, \hat{\Phi}^{-1}\right) \tilde{\Delta}\right\}$ are independent, that is, for any nonzero $q \in C^{n}, q^{*}[\tilde{N}-\tilde{M}]\left[I-\mathcal{F}_{L}\left(P, \hat{\Phi}^{-1}\right) \tilde{\Delta}\right] \neq 0$. We have

$$
\begin{aligned}
& q^{*}[\tilde{N}-\tilde{M}]\left[I-\mathcal{F}_{L}\left(P, \hat{\Phi}^{-1}\right) \tilde{\Delta}\right] \\
& =q^{*}[\tilde{N} \quad-\tilde{M}]\left(I-P_{11} \tilde{\Delta}\right) \\
& =q^{*}[\tilde{N} \quad-\tilde{M}]\left(I-\left[\begin{array}{c}
0 \\
r \tilde{M}^{-1}
\end{array}\right]\left[\begin{array}{ll}
r^{-1} \Delta_{N} & -r^{-1} \Delta_{M}
\end{array}\right]\right) \\
& =q^{*}\left[\begin{array}{cc}
\tilde{N} & -\tilde{M}
\end{array}\right]\left[\begin{array}{cc}
I & 0 \\
-\tilde{M}^{-1} \Delta_{N} & I+\tilde{M}^{-1} \Delta_{M}
\end{array}\right] \\
& =q^{*}\left[\tilde{N}+\Delta_{N} \quad-\left(\tilde{M}+\Delta_{M}\right)\right] \neq 0 .
\end{aligned}
$$

The last inequality follows from the fact that the matrix $\tilde{M}+$ $\Delta_{M}$ has full rank from our assumption that $\|\tilde{\Delta}\|<1$ and A1). This shows that $\mathcal{N}_{L}\left\{I-\mathcal{F}_{L}\left(P, \hat{\Phi}^{-1}\right) \tilde{\Delta}\right\}$ and $\mathcal{N}_{L}\left\{P_{12}\right\}$ are independent, but also that $\operatorname{dim}\left(\mathcal{N}_{L}\left\{I-\mathcal{F}_{L}\left(P, \hat{\Phi}^{-1}\right) \tilde{\Delta}\right\}\right)=p$. The latter implication follows from the inequality $\operatorname{dim}\left(\mathcal{N}_{L}\{I-\right.$ $\left.\left.\mathcal{F}_{L}\left(P, \hat{\Phi}^{-1}\right) \tilde{\Delta}\right\}\right) \leq n+p-\operatorname{dim}\left(\mathcal{N}_{L}\left\{P_{12}\right\}\right)=p$ and the inequality obtained above: $\operatorname{dim}\left(\mathcal{N}_{L}\left\{I-\mathcal{F}_{L}\left(P, \hat{\Phi}^{-1}\right) \tilde{\Delta}\right\}\right) \geq p$. But by Lemma 3, this implies that $\mathcal{N}_{L}\left\{I-\mathcal{F}_{L}\left(P, \hat{\Phi}^{-1}\right) \tilde{\Delta}\right\} \in$ $\mathcal{Y}$. Hence, $\exists U$ invertible, $Q$ such that

$\mathcal{N}_{L}\left\{I-\mathcal{F}_{L}\left(P, \hat{\Phi}^{-1}\right) \tilde{\Delta}\right\}=$ row span $\left(U P_{12}^{\dagger}+Q[\tilde{N}-\tilde{M}]\right)$

and therefore

$$
\begin{aligned}
& \left(U P_{12}^{\dagger}+Q[\tilde{N}-\tilde{M}]\right)\left[I-\mathcal{F}_{L}\left(P, \hat{\Phi}^{-1}\right) \tilde{\Delta}\right]=0 \\
& \left(P_{12}^{\dagger}-Q_{0}[\tilde{N}-\tilde{M}]\right)\left[I-\mathcal{F}_{L}\left(P, \hat{\Phi}^{-1}\right) \tilde{\Delta}\right]=0
\end{aligned}
$$

where $Q_{0}=-U^{-1} Q$.

2) $\Rightarrow 3)$ : Suppose that $\exists Q$ such that

$$
\left(P_{12}^{\dagger}-Q[\tilde{N}-\tilde{M}]\right)\left[I-\mathcal{F}_{L}\left(P, \hat{\Phi}^{-1}\right) \tilde{\Delta}\right]=0 .
$$

But $P_{12}^{\dagger}-Q\left[\begin{array}{ll}\tilde{N} & -\tilde{M}\end{array}\right]$ has full row rank $p$, hence $\operatorname{rank}\left\{I-\mathcal{F}_{L}\left(P, \hat{\Phi}^{-1}\right) \tilde{\Delta}\right\} \leq n$. 
With this lemma in hand, it is now possible to set up an optimization problem to find a $\tilde{\Delta}$ of norm less than one such that $\operatorname{rank}\left\{I-\mathcal{F}_{L}\left(P, \hat{\Phi}^{-1}\right) \tilde{\Delta}\right\} \leq n$.

Problem 9: Compute $\beta:=\inf \left\{\|\tilde{\Delta}\|: \operatorname{rank}\left\{I-\mathcal{F}_{L}(P\right.\right.$, $\left.\left.\left.\hat{\Phi}^{-1}\right) \tilde{\Delta}\right\} \leq n, \tilde{\Delta} \in C^{n \times(p+n)}\right\}$.

If the infimum $\beta$ is less than one, then the coprime factor model is consistent with the datum, otherwise it is not. Problem 9 is readily solved by a version of the Schmidt-Mirsky theorem [23].

Theorem 7 (Schmidt-Mirsky): Let $E \in C^{q \times r}, F \in C^{r \times q}$. Then for $i=1, \cdots, \min \{q, r\}$

$$
\inf \{\|E\|: \operatorname{rank}(I-F E) \leq r-i\}=\sigma_{i}(F)^{-1} .
$$

Furthermore, the infimum is achieved by a matrix $\hat{E}$ for which $\operatorname{rank}(I-F \hat{E})=r-i$.

The result of Theorem 7 tailored to our problem is just $\beta=\sigma_{p}\left[\mathcal{F}_{L}\left(P, \hat{\Phi}^{-1}\right)\right]^{-1}$. Hence the main lemma leading to our main consistency result, Theorem 3 , goes as follows.

Lemma 5: For a frequency $\omega$ and an invertible measurement datum $\Phi \in C^{p \times p}$ with $\hat{\Phi}-P_{22}$ also invertible, Problem 8 has a positive answer iff $\sigma_{p}\left[\mathcal{F}_{L}\left(P, \hat{\Phi}^{-1}\right)\right]^{-1}<1$.

Proof (Sufficiency): Suppose $\sigma_{p}\left[\mathcal{F}_{L}\left(P, \hat{\Phi}^{-1}\right)\right]^{-1}<1$. Then by Theorem 7, $\exists \tilde{\Delta} \in \mathcal{B} C^{n \times(p+n)}$ such that $\operatorname{rank}\{I-$ $\left.\mathcal{F}_{L}\left(P, \hat{\Phi}^{-1}\right) \tilde{\Delta}\right\}=n$. Moreover, $I-P_{11} \tilde{\Delta}$ is nonsingular since

$$
\left\|P_{11} \tilde{\Delta}\right\| \leq\left\|P_{11}|||\tilde{\Delta} \| \leq| r \mid \underline{\sigma}(\tilde{M})^{-1}<1\right.
$$

where the last inequality follows from A1). Hence by Lemma 4, $\hat{\Phi}-\mathcal{F}_{U}(P, \tilde{\Delta})=0$.

Necessity: Suppose that for $\tilde{\Delta} \in \mathcal{B} C^{n \times(p+n)}, \hat{\Phi}-$ $\mathcal{F}_{U}(P, \tilde{\Delta})=0$. Obviously, $I-P_{11} \tilde{\Delta}$ is nonsingular since $\|\tilde{\Delta}\|<1$. Thus by Lemma 4 , this is equivalent to $\operatorname{rank}\left\{I-\mathcal{F}_{L}\left(P, \hat{\Phi}^{-1}\right) \tilde{\Delta}\right\} \leq n$, which implies that $\beta<1$, i.e., $\sigma_{p}\left[\mathcal{F}_{L}\left(P, \hat{\Phi}^{-1}\right)\right]^{-1}<1$.

Finally, Lemma 5 and the boundary interpolation theorem (Theorem 6) can be used to prove the consistency result of Theorem 3.

Proof of Theorem 3 (Sufficiency): Suppose that $\forall i=$ $1, \cdots, N, \sigma_{p}\left[\mathcal{F}_{L}\left(P_{i}, \hat{\Phi}_{i}^{-1}\right)\right]^{-1}<1$. Then for each $i$, Lemma 5 says that $\exists \Delta_{i} \in \mathcal{B} C^{n \times(p+n)}$ making the perturbed CF model and the datum $\Phi_{i}$ consistent at frequency $\omega_{i}$. The unscaled $\Delta_{i}$ is such that $\| \Delta_{i}||<\left|r_{i}\right|$, and the boundary interpolation theorem (Theorem 6) used as in the proof of Theorem 2 says that there is a stable perturbation $\boldsymbol{\Delta} \in \mathcal{D}_{r}$ that interpolates the matrices $\left\{\Delta_{i}\right\}_{i=1}^{N}$ at $\left\{j \omega_{i}\right\}_{i=1}^{N}$.

Necessity: Suppose that for some $k, 1 \leq k \leq N$, $\sigma_{p}\left[\mathcal{F}_{L}\left(P_{k}, \hat{\Phi}_{k}^{-1}\right)\right]^{-1} \geq 1$. Then Lemma 5 says that $\Phi_{k}$ is inconsistent with the model at frequency $\omega_{k}$, i.e., the answer to Problem 3 is negative.

\section{APPENDIX C \\ PROOF OF THEOREM 4}

We must first prove Lemma 1. Let $q:=\left(\phi-V_{22}\right)^{-1}$.

Proof of Lemma 1 (Sufficiency): Suppose that $\operatorname{det}[I-$ $\left.\mathcal{F}_{L}\left(V, \phi^{-1}\right) \Delta\right]=0$. Then $\exists 0 \neq x \in C^{n+2}$ such that $x=V_{11} \Delta x+V_{12}\left(\phi-V_{22}\right)^{-1} V_{21} \Delta x$. Now $\left(I-V_{11} \Delta\right)$ is invertible by assumption, so we can write

$$
\begin{aligned}
\left(I-V_{11} \Delta\right) x & =q V_{12} V_{21} \Delta x \\
q^{-1} x & =\left(I-V_{11} \Delta\right)^{-1} V_{12} V_{21} \Delta x .
\end{aligned}
$$

Let $y \in C^{n+1}$ be defined as $y:=\Delta x$. Note that $y \neq 0$. Premultiply the last equation by $\Delta$ to get

$$
\begin{aligned}
q^{-1} y & =\Delta\left(I-V_{11} \Delta\right)^{-1} V_{12} V_{21} y \\
& =\left(I-\Delta V_{11}\right)^{-1} \Delta V_{12} V_{21} y
\end{aligned}
$$

where the last equation follows from the identity $\Delta(I-$ $\left.V_{11} \Delta\right)^{-1}=\left(I-\Delta V_{11}\right)^{-1} \Delta$. Let $z \in C$ be defined as $z:=V_{21} y \neq 0$, premultiply both sides of the last equation by $V_{21}$, and bring all the terms on the left-hand side to get

$$
\left[\phi-V_{22}-V_{21}\left(I-\Delta V_{11}\right)^{-1} \Delta V_{12}\right] z=0 .
$$

This last equation implies $\phi-\mathcal{F}_{U}(V, \Delta)=0$.

Necessity: Suppose that $\phi-\mathcal{F}_{U}(V, \Delta)=0$, that is

$$
\phi-V_{22}-V_{21} \Delta\left(I-V_{11} \Delta\right)^{-1} V_{12}=0 .
$$

Let $V_{12}^{\dagger}$ be the Moore-Penrose left-inverse of $V_{12}$. Then the last equation is equivalent to

$$
\left[q^{-1} V_{12}^{\dagger}-V_{21} \Delta\left(I-V_{11} \Delta\right)^{-1}\right] V_{12}=0
$$

or, equivalently, after a few algebraic manipulations (see [4] for more details)

$$
\left(V_{12}^{\dagger}-z^{*}\right)\{I-\underbrace{\left[V_{11}+V_{12}\left(1-\phi^{-1} V_{22}\right)^{-1} \phi^{-1} V_{21}\right]}_{\mathcal{F}_{L}\left(V, \phi^{-1}\right)} \Delta\}=0
$$

for some nonzero $z^{*} \in \mathcal{N}_{L}\left\{V_{12}\right\}$. By orthogonality of $V_{12}^{\dagger}$ and $z^{*}$, this implies that $I-\mathcal{F}_{L}\left(V, \phi^{-1}\right) \Delta$ is singular.

Definition 1: Let $U:=\left[\begin{array}{ll}U_{11} & U_{12} \\ U_{21} & U_{22}\end{array}\right]$ be complex and let $\tilde{\Delta}_{s} \in \Gamma$ have the same dimensions as $U_{11}^{T}$. Then the LFT $\mathcal{F}_{U}\left(U, \tilde{\Delta}_{s}\right)$ is well-posed if $I-U_{11} \tilde{\Delta}_{s}$ is invertible.

The following two lemmas are easily proven.

Lemma 6: The LFT $\mathcal{F}_{U}\left(U, \tilde{\Delta}_{s}\right)$ is well-posed $\forall \tilde{\Delta}_{s} \in \mathcal{B} \Gamma$ iff $\mu_{\Gamma}\left(U_{11}\right) \leq 1$.

Lemma 7: The LFT $\mathcal{F}_{U}\left(V, \tilde{\Delta}_{s}\right)$ is well-posed $\forall \tilde{\Delta}_{s} \in \mathcal{B} \Gamma$.

Introduce the normalized perturbations $\tilde{\Delta}_{i}:=\tilde{\boldsymbol{\Delta}}\left(j \omega_{i}\right)$ and $\tilde{w}_{a i}:=L_{a}^{-1} w_{a i}$. For each $\omega_{i}$, the consistency equation to be satisfied by admissible normalized perturbation and noise of norm less than one is just (13) at $\omega=\omega_{i}$. This equation can be rewritten in the form of (15), i.e., in LFT form with $\phi_{i}^{-1}$ as a feedback

$$
1-\phi_{i}^{-1} \mathcal{F}_{U}\left[\mathbf{V}\left(j \omega_{i}\right), \tilde{\Delta}_{s i}\right]=0 .
$$

Thus for each frequency $\omega_{i}$, a problem equivalent to the consistency problem can be formulated.

Problem 10: Does there exist a perturbation $\tilde{\Delta}_{s i}$ of norm less than one that satisfies (29)?

This problem can be readily solved by computing $\mu_{\Gamma}\left\{\mathcal{F}_{L}\left[\mathbf{V}\left(j \omega_{i}\right), \phi_{i}^{-1}\right]\right\}$ and checking that its value is larger than one (the MATLAB ${ }^{\mathrm{TM}} \mu$-Analysis and Synthesis toolbox [1] contains algorithms to compute $\mu$ ). That is, for each $\omega_{i}$, the consistency problem can be interpreted as a $\mu$ test for nonrobustness of the feedback interconnection in Fig. 6. This result is summarized in the following lemma. 
Lemma 8: We are given a frequency $\omega \in R_{+}$and a nonzero complex measurement $\phi$. Then $\exists \tilde{\Delta}_{s} \in \mathcal{B} \Gamma$ such that $\phi-\mathcal{F}_{U}\left(V, \tilde{\Delta}_{s}\right)=0$ iff $\mu_{\Gamma}\left[\mathcal{F}_{L}\left(V, \phi^{-1}\right)\right]>1$.

Proof: We have $\mu_{\Gamma}\left[\mathcal{F}_{L}\left(V, \phi^{-1}\right)\right]>1$ iff there exists $\tilde{\Delta}_{s} \in \mathcal{B} \Gamma$ such that $I-\mathcal{F}_{L}\left(V, \phi^{-1}\right) \tilde{\Delta}_{s}$ is singular. By Lemma 7 on well-posedness of $\mathcal{F}_{U}\left(V, \tilde{\Delta}_{s}\right), I-V_{11} \tilde{\Delta}_{s}$ is nonsingular, so Lemma 1 applies and we have that $I-$ $\mathcal{F}_{L}\left(V, \phi^{-1}\right) \tilde{\Delta}_{s}$ is singular iff $\phi-\mathcal{F}_{U}\left(V, \tilde{\Delta}_{s}\right)=0$.

We can now proceed with the proof of Theorem 4

Proof of Theorem 4 (Necessity): Suppose that Problem 4 has a positive answer, i.e., there exist $\boldsymbol{\Delta} \in \mathcal{D}_{r}$ and complex noises $\left\{w_{a i}\right\}_{i=1}^{N}$ in $\mathcal{W}$ such that $\mathcal{F}_{U}\left(V_{i}, \tilde{\Delta}_{s i}\right)=\phi_{i}$, $i=1, \cdots, N$. Then $\left\|\tilde{\Delta}_{s i}\right\|<1$, and by Lemma 8 , $\mu_{\Gamma}\left\{\mathcal{F}_{L}\left[\mathbf{V}\left(j \omega_{i}\right), \phi_{i}^{-1}\right]\right\}>1$, for $i=1, \cdots, N$.

Sufficiency: Suppose that $\mu_{\Gamma}\left\{\mathcal{F}_{L}\left[\mathbf{V}\left(j \omega_{i}\right), \phi_{i}^{-1}\right]\right\}>1$, for $i=1, \cdots, N$. Then by Lemma 8 , there exist $\left\{\tilde{\Delta}_{i}\right\}_{i=1}^{N}$, $\left\{\tilde{w}_{a i}\right\}_{i=1}^{N}$ of norm all less than one, such that the consistency equation (29) holds for each $i$. If we let $\Delta_{i}:=\mathbf{r}\left(j \omega_{i}\right) \tilde{\Delta}_{i}$ and $w_{a i}=L_{a} \tilde{w}_{a i}$ (the unscaled perturbations and noises), it follows that for each $i, \| \Delta_{i}||<\left|\mathrm{r}\left(j \omega_{i}\right)\right|$ and $w_{a i} \in \mathcal{W}$. Finally, the boundary interpolation theorem (Theorem 6) is used to show that there exists a $\Delta \in \mathcal{D}_{r}$ that interpolates the matrices $\left\{\Delta_{i}\right\}_{i=1}^{N}$ at $\left\{j \omega_{i}\right\}_{i=1}^{N}$. Hence, the corresponding perturbed plant $\mathbf{g}_{p}$ belongs to $\mathcal{P}$, and Problem 4 has a positive answer.

\section{REFERENCES}

[1] G. J. Balas, J. C. Doyle, K. Glover, A. Packard, and R. S. Smith, $\mu$-Analysis and Synthesis Toolbox: User's Guide. Natick, MA: Mathworks, 1991.

[2] J. A. Ball, I. Gohberg, and L. Rodman, Interpolation of Rational Matrix Functions. Operator Theory: Advances and Applications, vol 45. Boston, MA: Birkhäuser Verlag, 1990.

[3] D. S. Bayard, Y. Yam, and E. Mettler, "A criterion for joint optimization of identification and robust control," IEEE Trans. Automat. Contr., vol. 37, pp. 986-991, July 1992.

[4] B. Boulet, "Modeling and robust control of large flexible space structures," Ph.D. dissertation, Dept. Electrical and Computer Engineering, Univ. Toronto, 1996

[5] B. Boulet, B. A. Francis, P. C. Hughes, and T. Hong, "Uncertainty modeling and experiments in $\mathcal{H}_{\infty}$ control of large flexible space structures," IEEE Trans. Contr. Syst. Technol., vol. 5, no. 5, pp. 504-519, Sept. 1997.

[6] J. Chen, "Frequency-domain tests for validation of linear fractional uncertain models," IEEE Trans. Automat. Contr., vol. 42, pp. 748-760, June 1997.

[7] J. Chen, C. N. Nett, and M. K. H. Fan, "Worst-case system identification in $\mathcal{H}_{\infty}$ : Validation of a priori information, essentially optimal algorithms, and error bounds," in Proc. American Control Conf., 1992, pp. 251-257.

[8] P. H. Delsarte, Y. Genin, and Y. Kamp, The Nevanlinna-Pick problem for matrix-valued functions, SIAM J. Appl. Math., vol. 36, no. 1, pp. 47-61, Feb. 1979.

[9] J. C. Doyle, "Analysis of feedback systems with structured uncertainties," Proc. Inst. Elec. Eng., Nov. 1982, vol. 129, pt. D, no. 6, pp. 242-250.

[10] I. P. Fedčina, "A criterion for the solvability of the Nevanlinna-Pick tangent problem," Mat. Issled., vol. 7 (vyp. 4), no. 26, pp. 213-227, 1972.

[11] M. Green and D. J. N. Limebeer, Linear Robust Control, Information and System Sciences Series. Englewood Cliffs, NJ: Prentice-Hall, 1995.

[12] G. Gu, D. Xiong, and K. Zhou, "Identification in $\mathcal{H}_{\infty}$ using Pick's interpolation," Syst. Contr. Lett., vol. 20, pp. 263-272, Nov. 1993.

[13] R. A. Horn and C. R. Johnson, Matrix Analysis. Cambridge, U.K.: Cambridge Univ. Press, 1990
[14] P. P. Khargonekar and A. Tannenbaum, "Non-Euclidean metrics and the robust stabilization of systems with parameter uncertainty," IEEE Trans. Automat. Contr., vol. AC-30, pp. 1005-1013, Oct. 1985.

[15] R. L. Kosut, M. K. Lau, and S. P. Boyd, "Set-membership identification of systems with parametric and nonparametric uncertainty," IEEE Trans. Automat. Contr., vol. 37, pp. 929-941, July 1992.

[16] A. P. Low, G. O. Corrêa, and I. Postlethwaite, "Estimation of uncertainty bounds for robustness analysis," Proc. Inst. Elec. Eng., vol. 134, pt. D, 1987.

[17] D. C. McFarlane and K. Glover, "Robust controller design using normalized coprime factor plant descriptions," Lecture Notes in Control and Information Sciences. New York: Springer-Verlag, 1990.

[18] M. R. Michez, "Identification of a single and dual link flexible system," Master's thesis, Department of Electrical and Computer Engineering, Univ. Toronto, 1995

[19] M. Newlin and R. S. Smith, "Model validation and generalized $\mu$," in Proc. Conf. Decision and Control, Brighton, U.K., 1991, pp. 1257-1258.

[20] K. Poolla, P. P. Khargonekar, A. Tikku, J. Krause, and K. Nagpal, "A time-domain approach to model validation," IEEE Trans. Automat. Contr., vol. 39, pp. 951-959, May 1994.

[21] K. R. Popper, "The logic of scientific discovery," Basic Books. Toronto, Canada: Univ. Toronto Press, 1959.

[22] R. S. Smith and J. C. Doyle, "Model validation: A connection between robust control and identification," IEEE Trans. Automat. Contr., vol. 37, pp. 942-952, July 1992.

[23] G. W. Stewart and J. G. Sun, Matrix Perturbation Theory. San Diego, CA: Academic, 1990.

[24] M. Vidyasagar, Control System Synthesis: A Coprime Factorization Approach. Cambridge, MA: MIT Press, 1985.

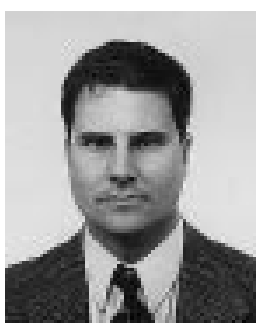

Benoit Boulet (S'88-M'92) was born in Saint-Tite, Québec, Canada in 1967. He received the B.A.Sc. degree from Université Laval, Québec, in 1990, the Master of Engineering degree from McGil University, Montréal, in 1992, and the Ph.D. degree from the University of Toronto in 1996, all in electrical engineering.

He worked for Hatch Associates Ltd. as Manager of the Advanced Control and Optimization group until October of 1998. He is a registered Professional Engineer in the provinces of Ontario and Québec. He is currently an Assistant Professor in the Department of Electrical and Computer Engineering at McGill University. His research interests include industrial process control, $\mathcal{H}_{\infty}$ control theory, robust control of large flexible structure, model validation for robust control, digital filter design based on Nevanlinna-Pick interpolation theory, and fuzzy logic control.

Dr. Boulet was the recipient of an NSERC postgraduate scholarship, an FCAR scholarship, a University of Toronto Open Fellowship, and a W. C. Sumner Fellowship during his graduate studies.

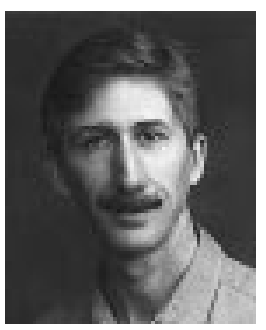

Bruce A. Francis (S'73-M'75-SM'85-F'88) was born in Toronto, Canada. He received the B.A.Sc. and M.Eng. degrees in mechanical engineering and the $\mathrm{Ph} . \mathrm{D}$. degree in electrical engineering from the University of Toronto in 1969, 1970, and 1975, respectively.

He has held research and teaching positions at the University of California, Berkeley, the University of Cambridge, Concordia University, McGill University, Yale University, the University of Waterloo, Caltech, and the University of Minnesota. He is presently a Professor of Electrical and Computer Engineering at the University of Toronto. He has coauthored three books, including Optimal Sampled-Data Control Systems, with T. Chen (1995). His current research interests include digital control and signal processing.

Dr. Francis was a corecipient of two Outstanding Paper Awards for papers appearing in the IEEE Transactions on Automatic Control and a corecipient of the 1991 IEEE W. R. G. Baker Prize Award. 